\title{
Multi-material topology optimization considering interface behavior via XFEM and level set method
}

\author{
Pai Liu, Yangjun Luo, Zhan Kang*
}

State Key Laboratory of Structural Analysis for Industrial Equipment, Dalian University of Technology Dalian 116024, China

\begin{abstract}
In most of existing topology optimization studies of multi-material structures, the interface of different materials was assumed to be perfectly bonded. Optimal design based on the perfectinterface assumption may introduce the risk of failure caused by interface debonding. This paper presents an efficient multi-material topology optimization strategy for seeking the optimal layout of structures considering the cohesive constitutive relationship of the interface. Based on the color level set method to describe the topology and the interface, the interface behavior is simulated by combining the extended finite element method (XFEM) and the cohesive model on fixed mesh. This enables modeling of possible separation of material interfaces, and thus provides a more realistic model of multi-material structures. Furthermore, this interface modeling technique avoids the difficulty of re-meshing when tracking the moving cohesive interface positions during the optimization process. In the topology optimization model, the normal velocities defined on the level set points are considered as design variables. In conjunction with the adjoint-variable sensitivity analysis, these design variables are updated by using the mathematical programming approach and then used to interpolate the boundary velocities. These boundary velocities are extrapolated to the whole domain with the fast marching method and used to advance the structural boundary through the Hamilton-Jacobi equation. This topology optimization technique can handle multiple constraints easily in the framework of level set method and at the same time preserve the signed distance property of the level set functions. Two numerical examples are given to demonstrate the effectiveness of the present method. It is also revealed that the optimal design considering interface behavior may exhibit tension/compression non-symmetric topology, in which material interfaces mainly undergo compression.
\end{abstract}

Keywords: Topology optimization; multi-material; interface debonding; cohesive model; XFEM; level set.

* Corresponding author. Email: zhankang@dlut.edu.cn; Tel. +86 41184706067 


\section{Introduction}

Since the seminal work of Bendsøe and Kikuchi on the homogenization method [1], the field of topology optimization has been developed rapidly. Nowadays, there are generally three kinds of topology optimization methods, namely the Solid Isotropic Material with Penalization (SIMP) method [2-4], the level set method [5, 6], and the Evolutionary Structural Optimization (ESO) method [7]. The details of three methods is discussed in the review papers [8-11].

As the technique of topology optimization develops and the demand for multi-functional design increases, the research on basic topology optimization methods have gradually turned to multi-phase materials from single-phase material. In the material density-based framework, Sigmund and Torquato [12] proposed a three-phase materials interpolation model for the stiffness and thermal strain coefficient tensors of extreme thermal expansion structures. This method was also extended to the microstructural design for extremal bulk modulus [13]. Further, Stegmann and Lund [14] employed a similar material interpolation model to the topology optimization of a composite shell structure, in which different fiber angles are treated as different phases of materials. To ensure that only one material is chosen at each point, Hvejsel and Lund [15] introduced a large number of constraints in density-based multi-material topology optimization. Based on a model called "shape function with penalization", Bruyneel [16] used two design variables to interpolate four kinds of material sections in the topology optimization of composite materials. Further, Gao et. al. [17] proposed a bi-value coding parameterization scheme to deal with a larger number of candidate orientations in discrete material optimization of composite laminates. Yin and Ananthasuresh [18] proposed a peak function model to interpolate multi-materials by using a single set of design variables. Radman et. al. [19] extended the Bi-directional Evolutionary Structural Optimization (BESO) method to the topological design of microstructure composed of multi-phase materials for maximum stiffness and thermal conductivity.

Level set methods offer a clear description of the design's boundary while optimization. In this framework, a so-called color level set method was first proposed by Wang and Wang [20] in 2004 to design a structure that is composed of $2^{l}$ phases of materials with $l$ level set functions. Since then, the color level set has been widely employed for the multi-material 
topology design of compliant mechanisms [21] and heat conduction problems [22]. Mei and Wang [23] and Wang et. al. [24] proposed different methods to describe $l$ phases of materials with $l-1$ level set functions. Luo et. al. [25] presented an indicator function with expression that is composed of piecewise constant level set functions for the optimization of piezoelectric actuators.

When considering a structure that consists of multi-phase materials, the behavior of the interface between different material phases plays a key role on the structural integrity and durability. The failure of a bonding interface may lead to collapse of a structure. However, all the above mentioned methods assume that the interfaces between the multi-materials are perfectly bonded. Little research has been done to consider to the performance of the material interfaces in structural optimization. Liu et. al. [26] designed the shape of the interface between two-phase materials with a non-parametric shape optimization method, in which the positions of the nodes are treated as design variables. Lawry and Maute [27] used a parameterized level set function to describe the sliding contact surfaces between two-phase materials and optimizes the shapes of these contact surfaces. Hilchenbach and Ramm [28] considered the damage of the interface between the matrix and the inclusion by combining the XFEM and a cohesive model, and optimized the size/position of inclusions with user-specified shapes to improve the ductility of the design using parametric optimization techniques. In structural optimization, with the ability to change the shape and layout of the materials simultaneously, topology optimization is seen as a more efficient tool. To the authors' knowledge, the issue of multi-materials topology optimization considering interface behavior has been not studied in literature.

In this study, we consider the maximum stiffness topology optimization problem with multiphase materials. The color level set method is adopted to represent the topology of the design, as well as to define the bonding interface. We equip the bonding interfaces between different phases of materials with a cohesive constitutive law. This enables a more realistic description of the interface, which may have separation and traction when the structure is loaded. In combination with the cohesive model, the XFEM is used to compute the displacement field including discontinuities on fixed mesh with arbitrary interface shape. In contrast to the finite element method with interface elements, in which the mesh needs to conform to the geometry 
of the interfaces, the XFEM avoids the difficulties of re-meshing and refinement (especially when the geometry is complex), and provides reliable results at a lower computational cost. Since the structural response is nonlinear in this setting, it is solved with an incremental solution procedure. In each incremental step, a Newton-Raphson (N-R) method is used to find the equilibrium point iteratively. In the optimization model, the objective function is the stored energy of a structure, and the normal velocities of the level set points are considered as design variables. An analytical sensitivity scheme for computing the derivatives of the objective function with respect to these design variables is derived with the adjoint method. The design variables are updated by the mathematical programing algorithm MMA (the method of moving asymptotes) [29]. After the normal velocities of the boundary points are interpolated using the design variables, they are then extended to the whole design domain with the fast marching method [30]. The Hamilton-Jacobi equation is solved to advance the level set functions and update the structural topology/shape. As compared with the steepest descent direction-based standard implementation of the level set method, this approach enables multiple constraints to be conveniently treated with a generic optimizer.

The organization of the rest of this paper is as follows: The next section introduces the interface modeling techniques for multi-material topology optimization problems, including the cohesive model and the extended finite element method (XFEM). Further, these techniques are incorporated into the color level set method to predict mechanical behaviors of material interfaces evolving with updated design during optimization. In Section 3, the mathematical formulation of the considered topology optimization problem and the solution procedures are elaborated. Section 4 is devoted to derivation of the shape sensitivity analysis scheme. Numerical examples are presented in Section 5 to demonstrate the effectiveness of the proposed method. Finally, conclusions and discussions are given in Section 6.

\section{Interface modelling in multi-material topology optimization}

A schematic illustration of the interface analysis problem for a structure composed of two materials and the void phase is depicted in Fig. 1. To optimize such a structure, we employ the 
color level set method to describe the shape/topology of the material boundaries, including the bonding interfaces. The cohesive model, in conjunction with the XFEM, which has been widely used in fracture mechanics analysis (e.g. [31-33]), is also used here to compute the structural response with consideration of the interface behavior.

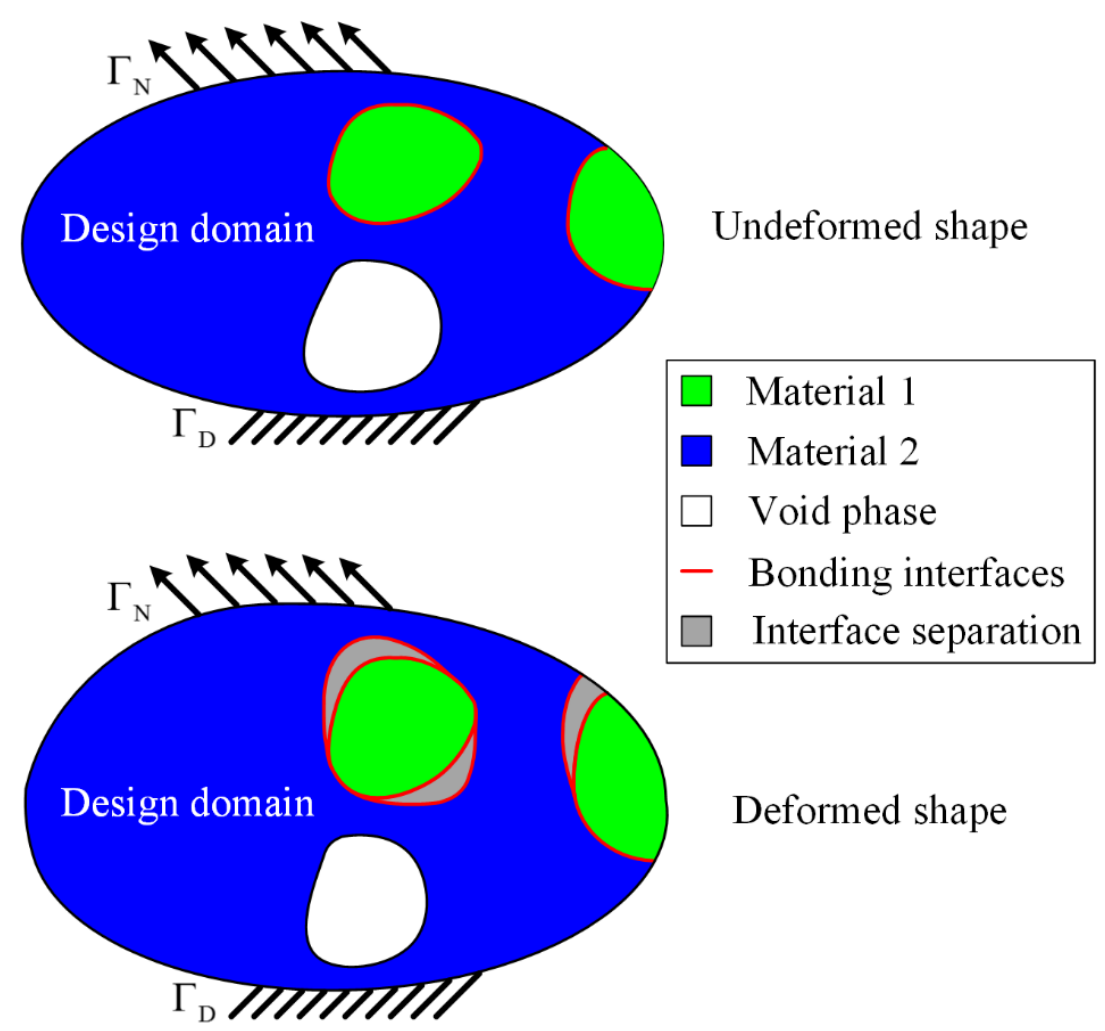

Fig. 1 A schematic illustration of the analysis problem

\subsection{Cohesive model for interface}

There are generally two types of cohesive models, the elastic-softening cohesive model and the general cohesive model [34]. The former assumes the behavior of the interface to be linear elastic before occurrence of the maximum traction and then adopts a nonlinear model to further describe the damage process of the interface. The latter uses a nonlinear smooth function which specifies both the loading and damage behavior of the interface.

The advantage of the general cohesive model is that it can capture the damage behavior automatically after the traction achieves the maximum value without assuming a damage 
initialization criterion. We therefore adopt the general cohesive model proposed in [35], which takes into account the tractions in both the normal and the sliding direction of an interface. Here we confine our problem to the cases of monotonic loading and small deformations.

The cohesive constitutive relationship in $2 \mathrm{D}$ problems reads

$$
\mathbf{t}=\frac{t_{\text {equ }}}{\delta_{\text {equ }}}\left(\beta^{2} \boldsymbol{\delta}_{\mathrm{s}}+\boldsymbol{\delta}_{\mathrm{n}}\right)
$$

where $\mathbf{t}$ is the traction vector, $\boldsymbol{\delta}_{\mathrm{s}}$ and $\boldsymbol{\delta}_{\mathrm{n}}$ are the separation vectors in the sliding direction and the normal direction of the interface, respectively; $t_{\text {equ }}$ and $\delta_{\text {equ }}$ denote the equivalent values of the traction and the separation on the interface, respectively:

$$
\begin{aligned}
& \delta_{\text {equ }}=\sqrt{\beta^{2}\left|\boldsymbol{\delta}_{\mathrm{s}}\right|^{2}+\left|\boldsymbol{\delta}_{\text {ne }}\right|^{2}}, \\
& t_{\text {equ }}=\sqrt{\beta^{-2}\left|\mathbf{t}_{\mathrm{s}}\right|^{2}+\left|\mathbf{t}_{\text {ne }}\right|^{2}},
\end{aligned}
$$

in which $\beta$ is a coefficient to tune the weight of the normal and sliding separations in the equivalent separation; $\boldsymbol{\delta}_{\mathrm{ne}}$ and $\mathbf{t}_{\mathrm{ne}}$ are the effective normal separation and traction vectors, respectively

$$
\begin{gathered}
\boldsymbol{\delta}_{\mathrm{ne}}= \begin{cases}\boldsymbol{\delta}_{\mathrm{n}} & \text { if } \boldsymbol{\delta}_{\mathrm{n}} \cdot \mathbf{n} \geq 0 \\
\mathbf{0} & \text { if } \boldsymbol{\delta}_{\mathrm{n}} \cdot \mathbf{n}<0\end{cases} \\
\mathbf{t}_{\mathrm{ne}}= \begin{cases}\mathbf{t}_{\mathrm{n}} & \text { if } \mathbf{t}_{\mathrm{n}} \cdot \mathbf{n} \geq 0 \\
\mathbf{0} & \text { if } \mathbf{t}_{\mathrm{n}} \cdot \mathbf{n}<0\end{cases}
\end{gathered}
$$

where $\mathbf{n}$ is the normal vector of the interface.

Note that Eqs. (2) and (3) ensure that the equivalent separation and traction only take the sliding and tension effects into account.

The equivalent traction $t_{\text {equ }}$ and the equivalent separation $\delta_{\text {equ }}$ yield the general bonding law proposed in [35]: 


$$
t_{\text {equ }}=e \sigma_{\text {c }} \frac{\delta_{\text {equ }}}{\delta_{\text {c }}} e^{-\delta_{\text {equ }} / \delta_{\mathrm{c}}},
$$

where $\sigma_{\mathrm{c}}$ is the maximum traction in the bonding law and $\delta_{\mathrm{c}}$ is the separation corresponding to $\sigma_{\mathrm{c}} ; e$ is the Euler's number. The fracture energy $G_{\mathrm{f}}$, which is defined as the integral of $t_{\text {equ }}$ on $\delta_{\text {equ }}$ from zero to infinity, can be calculated as $G_{\mathrm{f}}=e \sigma_{\mathrm{c}} \delta_{\mathrm{c}}$. Figure 2 shows the traction-separation relationship expressed by Eq. (6).

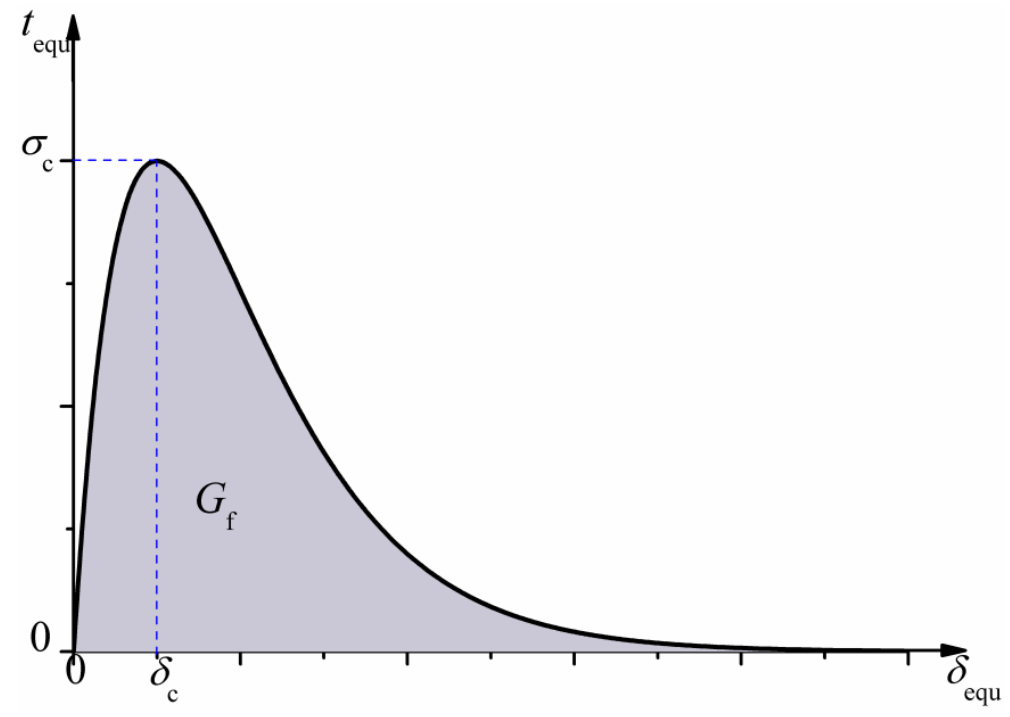

Fig. 2 A typical example of cohesive model

It can be seen from Eqs. (1)-(3) and Eq. (6) that $\boldsymbol{\delta}_{\mathrm{s}}$ and $\boldsymbol{\delta}_{\mathrm{n}}$ are coupled through the term $t_{\text {equ }} / \delta_{\text {equ }}$. For the cohesive constitutive law shown in Eq. (1), the differentiation of the traction in the interface's local coordinate system can be calculated using the tangent matrix $\mathbf{T}$ as

$$
\mathrm{d} \mathbf{t}_{\mathrm{loc}}=\mathbf{T} \mathrm{d} \boldsymbol{\delta}_{\text {loc }}
$$

in which $\mathbf{t}_{\mathrm{loc}}$ and $\boldsymbol{\delta}_{\mathrm{loc}}$ are the traction and separation vectors in the interface's local coordinate system. When the interface is under tension, the tangent matrix reads 


$$
\mathbf{T}=\left[\begin{array}{cc}
e \frac{\sigma_{\mathrm{c}}}{\delta_{\mathrm{c}}}\left[e^{-\delta_{\text {cqu }} / \delta_{\mathrm{c}}}-e^{-\delta_{\text {equ }} / \delta_{\mathrm{c}}} \frac{\delta_{\mathrm{n}}^{2}}{\delta_{\mathrm{c}} \delta_{\text {equ }}}\right] & e \frac{\sigma_{\mathrm{c}}}{\delta_{\mathrm{c}}}\left[-\beta^{2} e^{-\delta_{\text {equ }} / \delta_{\mathrm{c}}} \frac{\delta_{\mathrm{n}} \delta_{\mathrm{s}}}{\delta_{\mathrm{c}} \delta_{\text {equ }}}\right] \\
e \frac{\sigma_{\mathrm{c}}}{\delta_{\mathrm{c}}}\left[-\beta^{2} e^{-\delta_{\text {cqu }} / \delta_{\mathrm{c}}} \frac{\delta_{\mathrm{n}} \delta_{\mathrm{s}}}{\delta_{\mathrm{c}} \delta_{\text {equ }}}\right] & e \frac{\sigma_{\mathrm{c}}}{\delta_{\mathrm{c}}}\left[\beta^{2} e^{-\delta_{\text {equ }} / \delta_{\mathrm{c}}}-\beta^{4} e^{-\delta_{\text {equ }} / \delta_{\mathrm{c}}} \frac{\delta_{\mathrm{s}}^{2}}{\delta_{\mathrm{c}} \delta_{\text {equ }}}\right]
\end{array}\right],
$$

where $\delta_{\mathrm{n}}=\left|\boldsymbol{\delta}_{\mathrm{n}}\right|$ and $\delta_{\mathrm{s}}=\left|\boldsymbol{\delta}_{\mathrm{s}}\right|$. When the interface is under compression, we employ a large constant $d_{\mathrm{n}}$ to model the normal stiffness, and the coupling terms in the normal and shear directions in the tangent matrix become zero, which leads to

$$
\mathbf{T}=\left[\begin{array}{cc}
d_{\mathrm{n}} & 0 \\
0 & e \frac{\sigma_{\mathrm{c}}}{\delta_{\mathrm{c}}}\left[\beta^{2} e^{-\delta_{\text {cqu }} / \delta_{\mathrm{c}}}-\beta^{4} e^{-\delta_{\text {cqu }} / \delta_{\mathrm{c}}} \frac{\delta_{\mathrm{s}}^{2}}{\delta_{\mathrm{c}} \delta_{\text {equ }}}\right]
\end{array}\right]
$$

A schematic illustration of debonding of a 2D cohesive interface is depicted in Fig. 3.

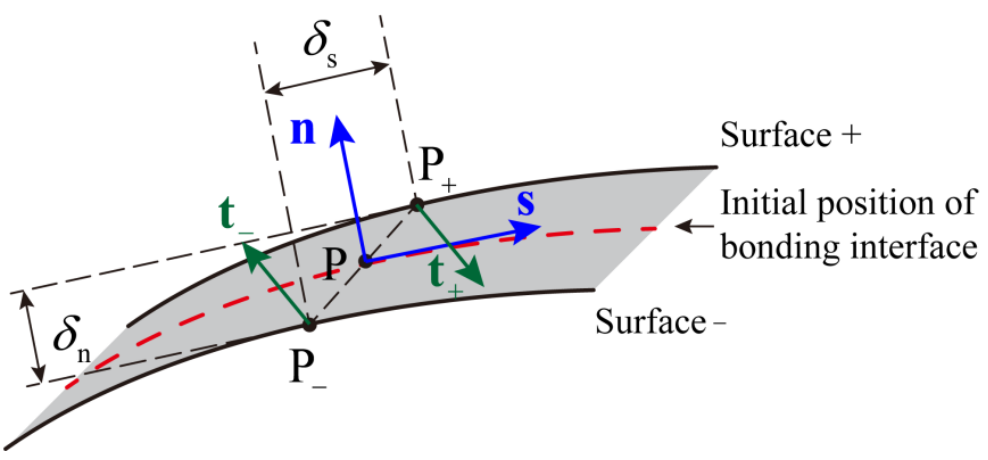

Fig. 3 Schematic illustration of debonding of a cohesive interface

In Fig. 3, $\mathbf{s}$ denotes the sliding direction vector of the bonding interface; $\mathrm{P}$ is a point on the bonding interface, and $\mathrm{P}_{+}$and $\mathrm{P}_{-}$respectively denote its position on surface + and surface - of the interface when debonding happens; $\mathbf{t}_{+}$and $\mathbf{t}_{-}$respectively denote the traction force on surface + and surface - .

\subsection{Extended finite element method}


The behavior of the interface obeys the cohesive law, which allows a certain separation of the interface. The XFEM describes this discontinuity of displacement field on a fixed mesh, by enriching a set of nodes with additional DOFs and interpolating them with discontinuous shape functions. For completeness, basic procedures of the XFEM for use in multi-material topology optimization are briefly introduced here.

An illustration of the enrichment strategy in XFEM is shown in Fig. 4. In this figure, the elements cut by the bonding interface, on which strong discontinuity of displacement exhibits, are enriched with additional DOFs. These additional DOFs enable a more accurate description of the opening of the bonding interface.

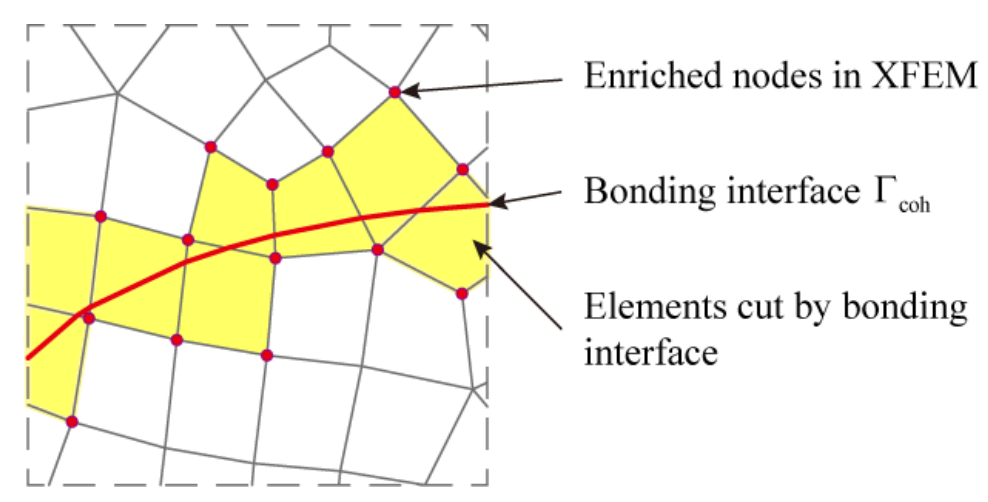

Fig. 4 XFEM enrichment at bonding interfaces

The displacement of any point $\mathbf{x}$ except the ones on the bonding interface can be expressed by

$$
\mathbf{u}(\mathbf{x})=\sum_{i \in \mathrm{D}} \mathrm{N}_{i}(\mathbf{x}) \mathbf{a}_{i}+\sum_{j \in \Gamma_{\text {coh }}} v(\mathbf{x}) \mathrm{N}_{j}(\mathbf{x}) \mathbf{b}_{j}
$$

where $\mathrm{D}$ is the design domain, $\Gamma_{\text {coh }}$ is the material interfaces on which the displacement is discontinuous; $\mathrm{N}_{i}$ and $\mathrm{N}_{j}$ are the conventional shape functions; $\mathbf{a}_{i}$ and $\mathbf{b}_{j}$ are the conventional and additional enriched displacement DOFs on the nodes, respectively; $v(\mathbf{x})$ is the discontinuous enrichment function. To rule out the influence of additional enriched DOFs 
outside the elements cut by the discontinuity, we express $v(\mathbf{x})$ in a shifted form as

$$
v(\mathbf{x})=h(\varphi(\mathbf{x}))-h\left(\varphi\left(\mathbf{x}_{j}\right)\right)
$$

in which $\varphi$ is the level set function on whose zero level set the discontinuity (bonding interface) is defined, $\mathbf{x}_{j}$ is the coordinate of node $j$ and $h(\varphi(\mathbf{x}))$ is defined by

$$
h(\varphi(\mathbf{x}))=\left\{\begin{array}{ll}
1 & \text { if } \varphi(\mathbf{x}) \geq 0 \\
-1 & \text { if } \varphi(\mathbf{x})<0
\end{array}\right\}
$$

For any point $\mathbf{x}$ on the bonding interface, the vector of separation (discontinuous displacement) $\mathbf{u}_{\text {disc }}$ in global coordinate system is interpolated as

$$
\mathbf{u}_{\mathrm{disc}}=2 \mathrm{~N}_{j}(\mathbf{x}) \mathbf{b}_{j}
$$

The separation vectors of the interface in the normal and sliding directions can be expressed as

$$
\begin{aligned}
& \boldsymbol{\delta}_{\mathrm{n}}=\left(\mathbf{u}_{\mathrm{disc}} \cdot \mathbf{n}\right) \otimes \mathbf{n}, \\
& \boldsymbol{\delta}_{\mathrm{s}}=\left(\mathbf{u}_{\mathrm{disc}} \cdot \mathbf{s}\right) \otimes \mathbf{s}
\end{aligned}
$$

It should be pointed that, in order to avoid the interference of the additional enriched DOFs for describing several bonding interfaces, we use an enrichment strategy mentioned in [36], which approximates the displacement field with a series of independent enriched DOFs.

\subsection{Interface modeling for multi-material topology optimization}

In the considered structural design problem with multi-phase materials, the color level set method is adopted to describe the topology evolution. This method can represent at most $2^{l}$ materials with / level set functions.

Taking a bi-material structural design problem as example, a schematic illustration of the topology representation model we use is depicted in Fig. 5. As can be seen, the design domain is divided into three types of material domains: $\Omega_{m} \quad(m=1,2,3)$. The characteristic 
functions $\chi_{m}$ in different material domains is expressed with two level set functions $\varphi_{1}$ and $\varphi_{2}$ as

$$
\begin{aligned}
& \chi_{1}=H\left(\varphi_{1}\right)\left(1-H\left(\varphi_{2}\right)\right) \\
& \chi_{2}=H\left(\varphi_{2}\right) \\
& \chi_{3}=\left(1-H\left(\varphi_{1}\right)\right)\left(1-H\left(\varphi_{2}\right)\right)
\end{aligned},
$$

where $H\left(\varphi_{l}\right)$ is the Heaviside function with the expression

$$
H\left(\varphi_{l}\right)=\left\{\begin{array}{l}
1 \text { if } \varphi_{l} \geq 0 \\
0 \text { if } \varphi_{l}<0
\end{array}\right\}
$$

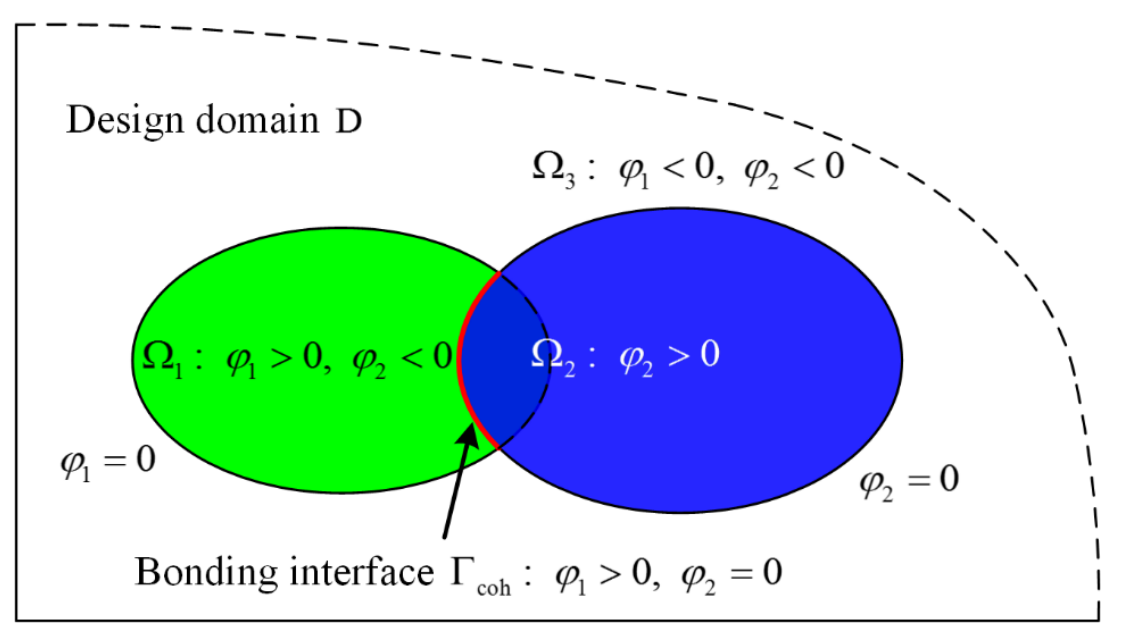

Fig. 5 A schematic illustration of level set representation of multi-material structure and material interface

The characteristic functions $\chi_{m}$ in Eq. (15) can ensure that only one material phase exists at each point. Typically, $\chi_{1}\left(\varphi_{1} \geq 0, \varphi_{2}<0\right)=1$ indicates material $1, \chi_{2}\left(\varphi_{2} \geq 0\right)=1$ indicates material 2 and $\chi_{3}\left(\varphi_{1}<0, \varphi_{2}<0\right)=1$ represents the void phase. In practice, we model the void phase with a weak material with very low stiffness. In Fig. 5, the interface is defined as the portion of zero level set of $\varphi_{2}$ on which $H\left(\varphi_{1}\right)$ equals 1 . The operation on the bonding 
interface can be easily accomplished with the Heaviside function and the Dirac delta function of $\varphi_{1}$ and $\varphi_{2}$.

In this study, the behavior of the interface is modeled with the combination of the level set representation, the cohesive model and the XFEM. A schematic illustration of this modelling technique is depicted in Fig. 6. In the figure, it can be seen that the level set points are uniformly distributed in the design domain. The level set functions $\varphi_{1}$ and $\varphi_{2}$ represent the boundary of the structure as well as the bonding interface implicitly. We note that the level set grid and the finite element mesh do not necessarily coincide. Therefore the values of $\varphi_{1}$ and $\varphi_{2}$ on the level set points are mapped onto the FEM nodes. On the FEM mesh, the structural boundary and the material interfaces can be found by linearly seeking the intersection points of the zero level sets of $\varphi_{1}, \varphi_{2}$ and the element edges, and thus a sequence of line segments of the material interfaces can be constructed. By enriching the nodes of the elements cut by the bonding interface with additional DOFs, the XFEM enables separation of the interface's surfaces, and thus can well describe interface behavior on the basis of the cohesive model.

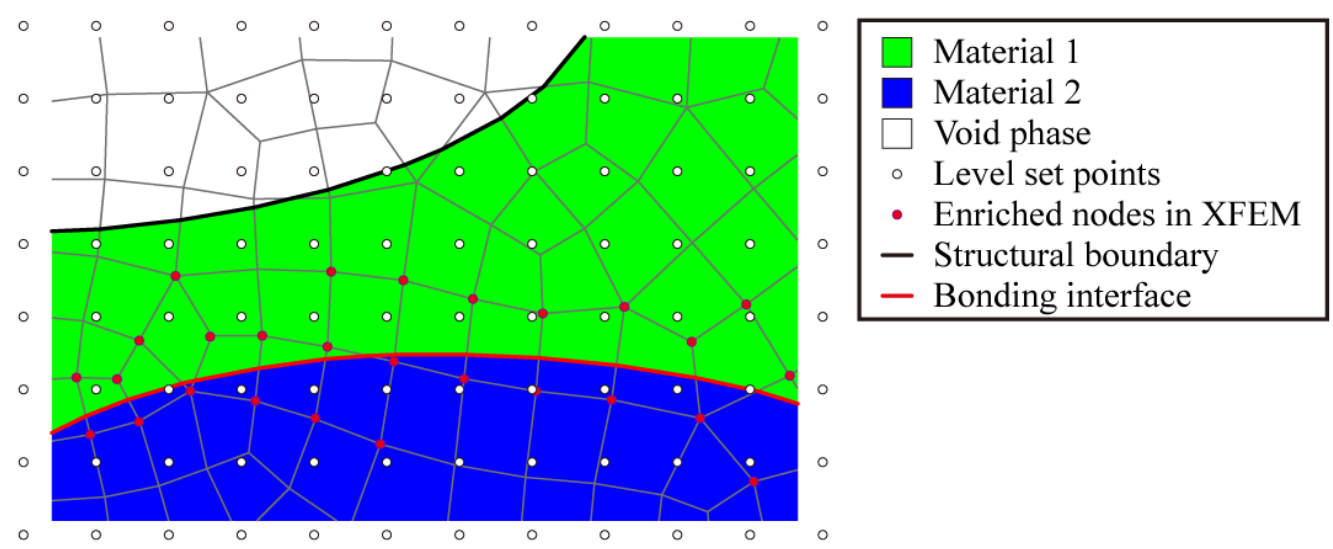

Fig. 6 Illustration of the interface modeling method for multi-material structure

\subsection{Nonlinear finite element analysis}

The virtual work principle of the equilibrium state of the studied structure reads 


$$
\int_{\mathrm{D}} \delta \boldsymbol{\varepsilon}^{\mathrm{T}} \boldsymbol{\sigma} \mathrm{d} \Omega+\int_{\Gamma_{\text {coh }}} \delta \mathbf{u}_{\mathrm{disc}}{ }^{\mathrm{T}} \mathbf{t} \mathrm{d} s=\int_{\mathrm{D}} \delta \mathbf{u}^{\mathrm{T}} \mathbf{f} \mathrm{d} \Omega+\int_{\Gamma_{\mathrm{N}}} \delta \mathbf{u}^{\mathrm{T}} \overline{\mathbf{t}} \mathrm{d} s
$$

where $\boldsymbol{\sigma}$ and $\boldsymbol{\varepsilon}$ are the stress vector and strain vector, respectively; $\mathbf{f}$ is the body force and $\overline{\mathbf{t}}$ is the external surface traction; $\delta \mathbf{u}, \delta \mathbf{u}_{\text {disc }}$ and $\delta \boldsymbol{\varepsilon}$ are the virtual displacement, virtual separation and virtual strain, respectively.

Based on Eqs. (10) and (13), the variation of the strain and the displacement discontinuity can be expressed as

$$
\begin{gathered}
\delta \boldsymbol{\varepsilon}=\mathbf{B} \delta \mathbf{a}+v(\mathbf{x}) \mathbf{B} \delta \mathbf{b}, \\
\delta \mathbf{u}_{\mathrm{disc}}=2 \mathbf{N} \delta \mathbf{b},
\end{gathered}
$$

where $\mathbf{N}$ is the shape function matrix, $\mathbf{B}$ is the strain-displacement matrix, $\delta \mathbf{a}$ and $\delta \mathbf{b}$ are the variations of the displacement vectors corresponding to the conventional and additional enriched DOFs, respectively.

By substituting Eqs. (18) and (19) into Eq. (17), one obtains the discrete form of the virtual work equation

$$
\left[\begin{array}{l}
\delta \mathbf{a} \\
\delta \mathbf{b}
\end{array}\right]^{\mathrm{T}}\left[\mathbf{f}_{\mathrm{int}}-\mathbf{f}_{\mathrm{ext}}\right]=0,
$$

where $\left[\begin{array}{ll}\delta \mathbf{a}^{\mathrm{T}} & \delta \mathbf{b}^{\mathrm{T}}\end{array}\right]^{\mathrm{T}} \equiv \delta \mathbf{d}$ denotes the variation of total displacement vector, $\mathbf{f}_{\mathrm{int}}$ is the internal force vector and $\mathbf{f}_{\mathrm{ext}}$ is the external force vector:

$$
\begin{gathered}
\mathbf{f}_{\text {int }}=\left[\begin{array}{c}
\int_{\mathrm{D}} \mathbf{B}^{\mathrm{T}} \boldsymbol{\sigma} \mathrm{d} \Omega \\
\int_{\mathrm{D}} v(\mathbf{x})^{\mathrm{T}} \mathbf{B}^{\mathrm{T}} \boldsymbol{\sigma} \mathrm{d} \Omega+\int_{\Gamma_{\mathrm{coh}}} 2 \mathbf{N}^{\mathrm{T}} \mathbf{t} \mathrm{d} s
\end{array}\right], \\
\mathbf{f}_{\mathrm{ext}}=\left[\begin{array}{c}
\left.\int_{\mathrm{D}} \mathbf{N}^{\mathrm{T}} \mathbf{f} \mathrm{d} \Omega+\int_{\Gamma_{\mathrm{N}}} \mathbf{N}^{\mathrm{T}} \overline{\mathbf{t}} \mathrm{d} s\right] \\
\mathbf{0}
\end{array}\right]
\end{gathered}
$$

Thus the residual force vector becomes $\mathbf{R}=\mathbf{f}_{\text {int }}-\mathbf{f}_{\text {ext }}$. 
As mentioned above, for each intermediate design, the structural boundary represented by the level set functions is found by locating the intersection points at the element boundaries and then approximated with line segments. A standard integration scheme in XFEM [37] is used, which decomposes the elements containing discontinuity into sub-elements for computing the Gaussian quadrature.

As the behavior of the bonding interface is nonlinear, an incremental solution procedure is adopted to solve this problem. By re-writing expression of $\mathbf{R}$ as $\mathbf{R}=\mathbf{f}_{\mathrm{int}}-\lambda \mathbf{f}_{\mathrm{ext}}$, in which $\lambda$ is the load factor, the residual force vector of increment $k+1$ can be expressed at the $k$ th increment as

$$
\mathbf{R}\left({ }^{k+1} \mathbf{d},{ }^{k+1} \lambda\right) \approx \mathbf{R}\left({ }^{k} \mathbf{d},{ }^{k} \lambda\right)+\mathbf{K}_{\mathrm{T}}\left({ }^{k} \mathbf{d}\right){ }^{k+1} \Delta \mathbf{d}-{ }^{k+1} \Delta \lambda \mathbf{f}_{\mathrm{ext}}=\mathbf{0},
$$

where $\mathbf{K}_{\mathrm{T}}=\partial \mathbf{R} / \partial \mathbf{d}$ is the tangent stiffness matrix, the superscripts $k+1$ and $k$ denote the incremental steps, ${ }^{k+1} \Delta \mathbf{d}={ }^{k+1} \mathbf{d}-{ }^{k} \mathbf{d}$ and ${ }^{k+1} \Delta \lambda={ }^{k+1} \lambda-{ }^{k} \lambda$.

In each incremental step, the N-R algorithm is employed to seek the equilibrium state in an iterative manner. Specifically, at the beginning of the N-R iterations for Eq. (23), the prescribed displacement boundary condition is imposed on the corresponding DOFs of ${ }^{k+1} \Delta \mathbf{d}$, then the $\mathrm{N}-\mathrm{R}$ iterations are repeated until convergence is achieved (when the norm of the residual force vector $\mathbf{R}\left({ }^{k+1} \mathbf{d}\right)$ becomes smaller than a specified tolerance value).

\section{Topology optimization formulation}

Without loss of generality, we hereafter consider topology optimization of structures composed of two materials for stiffness maximization. The mathematical programming method is used to obtain the velocities on the zero level sets of $\varphi_{1}$ and $\varphi_{2}$. An advantage of such an approach is that it can easily handle multiple constraints in the framework of level set method.

The design variables are two sets of scalar velocity values $\tilde{V}_{1 i}, \tilde{V}_{2 i}(i=1,2, \ldots, n)$ defined on the level set points. The normal evolution velocities of the points on the zero level sets of 
$\varphi_{1}$ or $\varphi_{2}$ can be obtained by bilinear interpolation of the design variables as

$$
V_{l \mathrm{n}}(\mathbf{x})=N_{i}(\mathbf{x}) \tilde{V}_{l i}, \quad l=1,2
$$

where $V_{l \mathrm{n}}(\mathbf{x})$ is the normal evolution velocity of $\mathbf{x}$ on the zero contour of $\varphi_{l} ; \tilde{V}_{l i}$ is the $i$ th design variable for $\varphi_{l}$.

The energy stored in a structure is defined as the sum of the strain energy stored in the materials and the work of the traction forces on the bonding interfaces. Thus the expression of the stored energy is

$$
f=\int_{\mathrm{D}}\left(\int_{\mathbf{0}}^{\bar{\varepsilon}} \boldsymbol{\sigma}^{\mathrm{T}} \mathrm{d} \boldsymbol{\varepsilon}\right) \mathrm{d} \Omega+\int_{\Gamma_{\text {coh }}}\left(\int_{\mathbf{0}}^{\overline{\mathrm{u}}_{\mathrm{disc}}} \mathbf{t}^{\mathrm{T}} \mathrm{d} \mathbf{u}_{\mathrm{disc}}\right) \mathrm{d} s,
$$

where $\overline{\boldsymbol{\varepsilon}}$ and $\overline{\mathbf{u}}_{\text {disc }}$ are the final strain in the material domain and the separation vector on the bonding interface, respectively.

In the case when only non-homogeneous Dirichlet boundary condition is applied and no external forces exist, the task of the design optimization problem is to maximize the stored energy $f$ (or to minimize $-f$ ) [38]. For regularization of the topology optimization problem, a length term of the bonding interface $\mathrm{P}$ together with its Lagrange multiplier (weight factor) $b$ are also added to the objective function to impose penalization of the total length of the material interface. Thus the objective function becomes

$$
J=-f+b \mathrm{P},
$$

where $b$ is a weighting factor and $\mathrm{P}$ is expressed by

$$
\mathrm{P}=\int_{\mathrm{D}} H\left(\varphi_{1}\right) \delta\left(\varphi_{2}\right)\left|\nabla \varphi_{2}\right| \mathrm{d} \Omega .
$$

With the constraint of equilibrium equation and two volume constraints, we formulate the topology optimization problem as 
Find $\quad \tilde{\mathbf{V}}_{\mathrm{n}}=\left\{\tilde{V}_{11}, \cdots, \tilde{V}_{1 n}, \tilde{V}_{21}, \cdots, \tilde{V}_{2 n}\right\}$

min. $J$

$$
\begin{array}{ll}
\text { s.t. } & \mathbf{R}\left({ }^{k} \mathbf{d}\left(\tilde{\mathbf{V}}_{\mathrm{n}}\right)\right)=\mathbf{0}, \quad k=1, \cdots, K, \\
& g_{1}=f_{\mathrm{V}_{1}}-f_{\overline{\mathrm{V}}_{1}} \leq 0, \\
& g_{2}=f_{\mathrm{V}_{2}}-f_{\overline{\mathrm{V}}_{2}} \leq 0, \\
& \tilde{V}_{\min } \leq \tilde{V}_{l i} \leq \tilde{V}_{\max }, \quad l=1,2 ; \quad i=1, \cdots, n
\end{array}
$$

where $f_{\overline{\mathrm{v}}_{1}}$ and $f_{\overline{\mathrm{v}}_{2}}$ are the given amount of volume fraction for material 1 and material 2 , respectively. $f_{\mathrm{V}_{1}}$ and $f_{\mathrm{V}_{2}}$ are the current material volume fractions, $K$ is the number of incremental steps, $n$ is the number of level set points, $\tilde{V}_{\min }$ and $\tilde{V}_{\max }$ are the lower and upper bound limits of the design variables. As seen in the interpolation relationship of Eq. (24), the design variables share the same lower and upper bound limit as the evolution velocities of the level set functions, which is confined by the CFL condition.

A mass constraint was suggested by Gao and Zhang [39] for topology optimization with multi-phase materials. However, in Eq. (28), the resource constraints still take the conventional form of material volume ratios.

For each intermediate design, the optimization problem in Eq. (28) is solved by the mathematical programming method MMA. Then the evolution velocities of the zero level sets of $\varphi_{1}$ and $\varphi_{2}$ are interpolated using the design variables as expressed by Eq. (24). These velocities are then extrapolated to the whole domain with the fast marching method.

The Hamilton-Jacobi equation with a diffusion term is used to advance level set functions and update the current design:

$$
\frac{\partial \varphi_{l}}{\partial t}+V_{l \mathrm{n}}\left|\nabla \varphi_{l}\right|-c \kappa_{l}\left|\nabla \varphi_{l}\right|=0, \quad l=1,2
$$

where $\kappa_{l}=\nabla \cdot\left(\nabla \varphi_{l} /\left|\nabla \varphi_{l}\right|\right)$ is the mean curvature of level set function $\varphi_{l}$, and $c$ is a userspecified diffusion coefficient. As can be seen from Eqs. (26) and (29), we regularize the optimization problem not only with a penalization of the length of the material interface, but also with a diffusion term in the Hamilton-Jacobi equation. 
As usual, in order to preserve the property of signed distance, we re-initialized the level set functions every 5 iterations during the process of topology optimization.

The flowchart of the topology optimization procedure is depicted in Fig. 7.

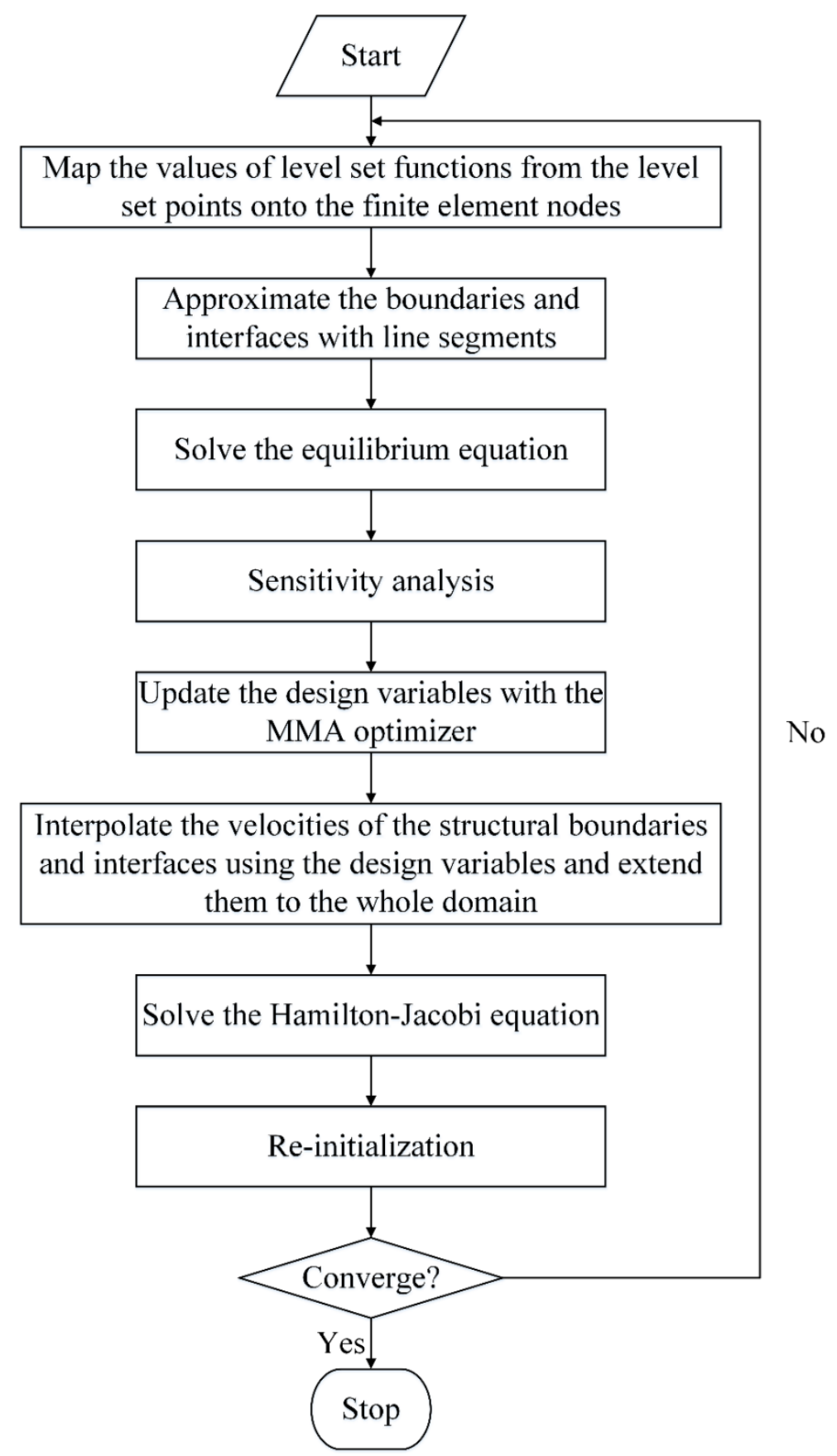

Fig. 7 Flowchart of the topology optimization procedures

\section{Sensitivity analysis}


We derive the sensitivities of the objective function with respect to the design variables for the equilibrium states computed with the incremental method. Rewriting Eq. (25) with a linear approximation between two successive convergence points, we have the following relation in matrix form

$$
\begin{aligned}
f= & \sum_{k=1}^{K}{ }^{k} f \\
= & \sum_{k=1}^{K}\left(\int_{\mathrm{D}} \sum_{m=1}^{3} \frac{1}{2}\left({ }^{k-1} \boldsymbol{\varepsilon}+{ }^{k} \boldsymbol{\varepsilon}\right){ }^{\mathrm{T}} \chi_{m} \mathbf{D}_{m}\left({ }^{k} \boldsymbol{\varepsilon}-{ }^{k-1} \boldsymbol{\varepsilon}\right) \mathrm{d} \Omega\right. \\
& \left.+\int_{\mathrm{D}} \frac{1}{2}\left({ }^{k-1} \mathbf{t}+{ }^{k} \mathbf{t}\right){ }^{\mathrm{T}}\left({ }^{k} \mathbf{u}_{\text {disc }}-{ }^{k-1} \mathbf{u}_{\text {disc }}\right) H\left(\varphi_{1}\right) \delta\left(\varphi_{2}\right)\left|\nabla \varphi_{2}\right| \mathrm{d} \Omega\right)
\end{aligned}
$$

where ${ }^{k} f$ represents the work of external force in the $k$ th incremental step, $\mathbf{D}_{m}$ is the elasticity matrix of material phase $m .{ }^{k} f$ can be further expressed as

$$
\begin{aligned}
{ }^{k} f= & \int_{\mathrm{D}}\left({ }^{k-1} \sigma+\frac{1}{2} \sum_{m=1}^{3}\left(\chi_{m} \mathbf{D}_{m}\right)^{k} \Delta \boldsymbol{\varepsilon}_{{ }^{k}}\right)^{\mathrm{T}}{ }^{k} \Delta \boldsymbol{\varepsilon}_{{ }^{k} \Delta \mathbf{u}} \mathrm{d} \Omega \\
& +\int_{\mathrm{D}}\left({ }^{k-1} \mathbf{t}+\frac{1}{2} \mathbf{G}^{\mathrm{T}}{ }^{k} \mathbf{T} \mathbf{G}^{k} \Delta \mathbf{u}_{\mathrm{disc}}\right)^{\mathrm{T}}{ }^{k} \Delta \mathbf{u}_{\mathrm{disc}} H\left(\varphi_{1}\right) \delta\left(\varphi_{2}\right)\left|\nabla \varphi_{2}\right| \mathrm{d} \Omega
\end{aligned}
$$

where $\mathbf{G}$ is a mapping matrix between the local coordinate system of the bonding interface and the global coordinate system; ${ }^{k} \Delta \mathbf{u}={ }^{k} \mathbf{u}-{ }^{k-1} \mathbf{u}$ is the displacement increment in the $k$ th incremental step; ${ }^{k} \Delta \boldsymbol{\varepsilon}_{{ } \Delta \mathbf{u}}$ represents the strain increment computed with ${ }^{k} \Delta \mathbf{u}$; and ${ }^{k} \Delta \mathbf{u}_{\text {disc }}$ is the increment of the interface separation.

By taking into account the incremental form of equilibrium equations, a Lagrangian function is constructed as

$$
L\left(\hat{\mathbf{u}}, \hat{\mathbf{w}}, \varphi_{1}, \varphi_{2}\right)=\sum_{k=1}^{K}\left(-{ }^{k} f\left(\hat{\mathbf{u}}, \varphi_{1}, \varphi_{2}\right)+{ }^{k} \alpha\left(\hat{\mathbf{u}}, \hat{\mathbf{w}}, \varphi_{1}, \varphi_{2}\right)\right)+b \mathrm{P}\left(\varphi_{1}, \varphi_{2}\right)
$$

where $\hat{\mathbf{u}}$ is the displacement field, which is composed of a continuous part $\mathbf{u}$ in the material domain and a discontinuous part $\mathbf{u}_{\text {disc }}$ on the bonding interfaces; $\hat{\mathbf{w}}$ is the adjoint displacement field corresponding to $\hat{\mathbf{u}} ;{ }^{k} \alpha$ is the incremental form of equilibrium equation of the $k$ th step multiplied by the adjoint variable and it has the expression 


$$
\begin{aligned}
{ }^{k} \alpha\left(\hat{\mathbf{u}}, \hat{\mathbf{w}}, \varphi_{1}, \varphi_{2}\right)= & \int_{\mathrm{D}}{ }^{k} \boldsymbol{\varepsilon}_{{ }^{k}}{ }^{\mathrm{T}} \sum_{m=1}^{3}\left(\chi_{m} \mathbf{D}_{m}\right)^{k} \Delta \boldsymbol{\varepsilon}_{{ }^{k}} \mathrm{du} \\
& \mathrm{d} \Omega \\
& +\int_{\mathrm{D}}{ }^{k} \mathbf{w}_{\mathrm{disc}}{ }^{\mathrm{T}} \mathbf{G}^{\mathrm{T}}{ }^{k} \mathbf{T} \mathbf{G}^{k} \Delta \mathbf{u}_{\mathrm{disc}} H\left(\varphi_{1}\right) \delta\left(\varphi_{2}\right)\left|\nabla \varphi_{2}\right| \mathrm{d} \Omega=0
\end{aligned}
$$

where $\mathbf{w}$ and $\mathbf{w}_{\text {disc }}$ are the adjoint displacements in the material domain and on the bonding interfaces, respectively.

The Fréchet derivative of the Lagrangian function $L$ with respect to $\hat{\mathbf{u}}$ in the incremental form reads

$$
\begin{aligned}
\left\langle\frac{\partial L}{\partial \hat{\mathbf{u}}}, \delta \hat{\mathbf{u}}\right\rangle & =\sum_{k=1}^{K} \frac{\partial L}{\partial^{k} \Delta \hat{\mathbf{u}}} \delta^{k} \Delta \hat{\mathbf{u}} \\
& =\sum_{k=1}^{K} \frac{\partial\left(-{ }^{k} f\right)}{\partial^{k} \Delta \hat{\mathbf{u}}} \delta^{k} \Delta \hat{\mathbf{u}}+\sum_{k=1}^{K} \frac{\partial^{k} \alpha}{\partial^{k} \Delta \hat{\mathbf{u}}} \delta^{k} \Delta \hat{\mathbf{u}} \\
& =\sum_{k=1}^{K}\left(\int_{\mathrm{D}} \delta^{k} \Delta \boldsymbol{\varepsilon}_{\delta^{k} \Delta \mathbf{u}}{ }^{\mathrm{T}}\left(-{ }^{k-1} \sigma-\sum_{m=1}^{3}\left(\chi_{m} \mathbf{D}_{m}\right)^{k} \Delta \boldsymbol{\varepsilon}_{{ }^{k} \Delta \mathbf{u}}+\sum_{m=1}^{3}\left(\chi_{m} \mathbf{D}_{m}\right)^{k} \boldsymbol{\varepsilon}_{{ }} \mathbf{w}\right) \mathrm{d} \Omega\right) \\
& +\sum_{k=1}^{K}\left(\int_{\mathrm{D}} \delta^{k} \Delta \mathbf{u}_{\mathrm{disc}}{ }^{\mathrm{T}}\left(-{ }^{k-1} \mathbf{t}-\mathbf{G}^{\mathrm{T} k} \mathbf{T} \mathbf{G}^{k} \Delta \mathbf{u}_{\mathrm{disc}}+\mathbf{G}^{\mathrm{T} k} \mathbf{T G}^{k} \mathbf{w}_{\mathrm{disc}}\right) H\left(\varphi_{1}\right) \delta\left(\varphi_{2}\right)\left|\nabla \varphi_{2}\right| \mathrm{d} \Omega\right)
\end{aligned}
$$

By rearranging the terms in Eq. (34), the adjoint equation is obtained as

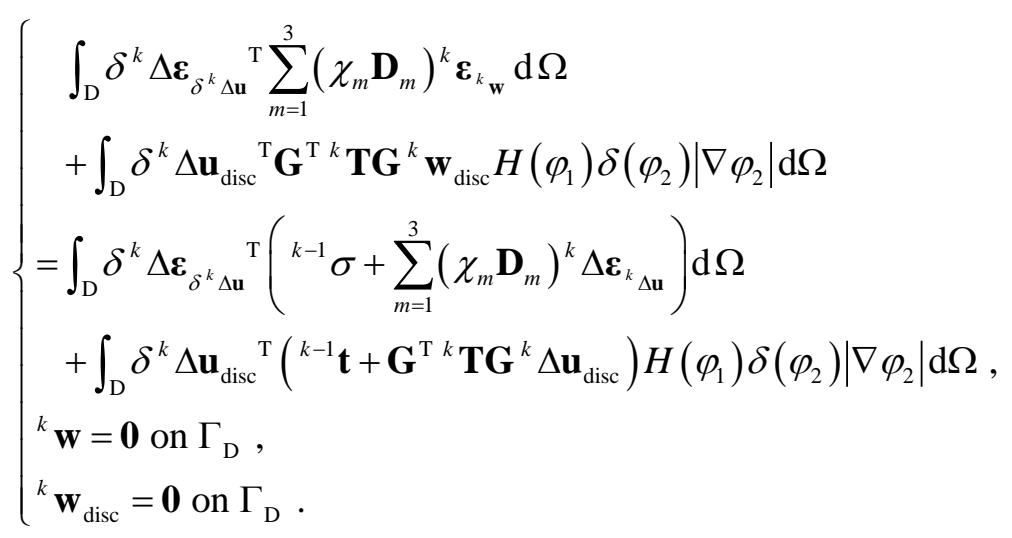

After solving Eq. (35) for $k=1, \ldots, K$, the adjoint variables ${ }^{k} \hat{\mathbf{w}}$ can be obtained. By inserting ${ }^{k} \hat{\mathbf{w}}$ into Eq. (32), the variation of the objective function due to the perturbation $\psi_{l}$ of the level set function $\varphi_{l}$ can be expressed as the Fréchet derivatives as

$$
\left\langle\frac{\partial L}{\partial \varphi_{1}}, \psi_{1}\right\rangle=\sum_{k=1}^{K}\left(\int_{D}\left(-\frac{1}{2}{ }^{k} \Delta \boldsymbol{\varepsilon}_{{ }_{\Delta} \Delta \mathbf{u}}+{ }^{k} \boldsymbol{\varepsilon}_{k_{\mathbf{w}}}\right)^{\mathrm{T}} \sum_{m=1}^{3}\left(\chi_{m} \mathbf{D}_{m}\right)_{, \varphi_{1}}{ }^{k} \Delta \boldsymbol{\varepsilon}_{{ }{ }_{\Delta \mathbf{u}}} \psi_{1} \mathrm{~d} \Omega\right)
$$




$$
\begin{aligned}
& \left\langle\frac{\partial L}{\partial \varphi_{2}}, \psi_{2}\right\rangle=\sum_{k=1}^{K}\left(\int_{D}\left(-\frac{1}{2}{ }^{k} \Delta \boldsymbol{\varepsilon}_{{ }_{\Delta \mathbf{u}}}+{ }^{k} \boldsymbol{\varepsilon}_{{ }}\right)^{\mathrm{T}}\right)^{\mathrm{T}} \sum_{m=1}^{3}\left(\chi_{m} \mathbf{D}_{m}\right)_{, \varphi_{2}}{ }^{k} \Delta \boldsymbol{\varepsilon}_{{ }{ }_{\Delta \mathbf{u}}} \psi_{2} \mathbf{d} \Omega \\
& -\int_{\mathrm{D}}\left(\left({ }^{k} \mathbf{w}_{\text {disc }}{ }^{\mathrm{T}} \mathbf{G}^{\mathrm{T} k}{ }^{k} \mathbf{T} \mathbf{G}^{k} \Delta \mathbf{u}_{\text {disc }}\right)_{, \mathrm{n}}+\kappa^{k} \mathbf{w}_{\text {disc }}{ }^{\mathrm{T}} \mathbf{G}^{\mathrm{T}}{ }^{k} \mathbf{T} \mathbf{G}^{k} \Delta \mathbf{u}_{\mathrm{disc}}\right) H\left(\varphi_{1}\right) \delta\left(\varphi_{2}\right) \psi_{2} \mathbf{d} \Omega \\
& +\int_{\mathrm{D}}\left(\left(\frac{1}{2}{ }^{k} \Delta \mathbf{u}_{\text {disc }}{ }^{\mathrm{T}} \mathbf{G}^{\mathrm{T} k}{ }^{k} \mathbf{T} \mathbf{G}^{k} \Delta \mathbf{u}_{\text {disc }}\right)_{, \mathrm{n}}+\frac{1}{2} \kappa^{k} \Delta \mathbf{u}_{\mathrm{disc}}{ }^{\mathrm{T}} \mathbf{G}^{\mathrm{T} k}{ }^{k} \mathbf{T} \mathbf{G}^{k} \Delta \mathbf{u}_{\mathrm{disc}}\right) H\left(\varphi_{1}\right) \delta\left(\varphi_{2}\right) \psi_{2} \mathbf{d} \Omega \\
& \left.+\int_{\mathrm{D}}\left(-\frac{1}{2}{ }^{k} \Delta \mathbf{u}_{\mathrm{disc}}+{ }^{k} \mathbf{w}_{\mathrm{disc}}\right)^{\mathrm{T}}\left(\mathbf{G}^{\mathrm{T} k} \mathbf{T} \mathbf{G}\right)_{, \varphi_{2}}{ }^{k} \Delta \mathbf{u}_{\mathrm{disc}} H\left(\varphi_{1}\right) \delta\left(\varphi_{2}\right)\left|\nabla \varphi_{2}\right| \psi_{2} \mathbf{d} \Omega\right) \\
& -b \int_{\mathrm{D}} \kappa H\left(\varphi_{1}\right) \delta\left(\varphi_{2}\right) \psi_{2} \mathrm{~d} \Omega
\end{aligned}
$$

On the right-hand side of Eq. (37), the directional derivatives $\left({ }^{k} \mathbf{w}_{\text {disc }}{ }^{\mathrm{T}} \mathbf{G}^{\mathrm{T}}{ }^{k} \mathbf{T} \mathbf{G}^{k} \Delta \mathbf{u}_{\mathrm{disc}}\right)_{\text {,n }}$ and $\left(\frac{1}{2}{ }^{k} \Delta \mathbf{u}_{\text {disc }}{ }^{\mathrm{T}} \mathbf{G}^{\mathrm{T} k} \mathbf{T G}^{k} \Delta \mathbf{u}_{\text {disc }}\right)_{\text {, }}$ are discarded because the variables in the bracket are only defined on the interface. Since the variation of the level set function is small between two successive optimization steps (restricted by the CFL condition), we also ignore the dependence of the normal direction vector $\mathbf{n}$ on the level set function $\varphi_{2}$. It follows that $\left(\mathbf{G}^{\mathrm{T} k} \mathbf{T G}\right)_{, \varphi_{2}}=0$ on the right-hand side of Eq. (37) because $\mathbf{G}_{, \varphi_{2}}=\mathbf{G}_{, \mathbf{n}} \mathbf{n}_{, \varphi_{2}}$ and ${ }^{k} \mathbf{T}_{, \varphi_{2}}={ }^{k} \mathbf{T}_{, \mathbf{n}} \mathbf{n}_{, \varphi_{2}}$ both vanish at this point.

Given $\varphi_{l}$ a finite perturbation $-V_{l \mathrm{n}}\left|\nabla \varphi_{l}\right|$, by substituting the expressions of $\chi_{m}$ in Eq. (15) into Eqs. (36) and (37), the change of the Lagrangian function can be written in the form of line integral on zero level sets of $\varphi_{1}$ and $\varphi_{2}$ as

$$
\begin{aligned}
\Delta L= & \sum_{k=1}^{K}\left(\int_{\varphi_{1}=0}\left(1-H\left(\varphi_{2}\right)\right)\left(\frac{1}{2}{ }^{k} \Delta \boldsymbol{\varepsilon}_{{ }^{k} \Delta \mathbf{u}}-{ }^{k} \boldsymbol{\varepsilon}_{k_{\mathbf{w}}}\right)^{\mathrm{T}} \mathbf{D}_{1}{ }^{k} \Delta \boldsymbol{\varepsilon}_{{ }} V_{\Delta \mathbf{u}} V_{1 \mathrm{n}} \mathrm{d} s\right. \\
& +\int_{\varphi_{2}=0}\left(\frac{1}{2}{ }^{k} \Delta \boldsymbol{\varepsilon}_{{ }^{k} \Delta \mathbf{u}}-{ }^{k} \boldsymbol{\varepsilon}_{{ }^{k} \mathbf{w}}\right)^{\mathrm{T}}\left(-H\left(\varphi_{1}\right) \mathbf{D}_{1}+\mathbf{D}_{2}\right){ }^{k} \Delta \boldsymbol{\varepsilon}_{{ }}{ }_{\Delta \mathbf{u}} V_{2 \mathrm{n}} \mathrm{d} s \\
& \left.-\int_{\varphi_{2}=0} \kappa\left(\frac{1}{2}{ }^{k} \Delta \mathbf{u}_{\mathrm{disc}}-{ }^{k} \mathbf{w}_{\text {disc }}\right)^{\mathrm{T}} \mathbf{G}^{\mathrm{T}}{ }^{k} \mathbf{T} \mathbf{G}^{k} \Delta \mathbf{u}_{\mathrm{disc}} V_{2 \mathrm{n}} H\left(\varphi_{1}\right) \mathrm{d} s\right) \\
& +b \int_{\varphi_{2}=0} \kappa H\left(\varphi_{1}\right) V_{2 \mathrm{n}} \mathrm{d} s
\end{aligned}
$$


in which $\Delta L$ denote the change of the Lagrangian function due to perturbations of $\varphi_{1}$ and $\varphi_{2}$.

As mentioned, the boundary velocities $V_{l \mathrm{n}}(l=1,2)$ are interpolated with the shape function using the design variables $\tilde{V}_{l i}$ defined on the level set points, and the objective function $J$ can be written as the sum of the current value of objective function $J_{0}$ and the change $\Delta J$ as $J=J_{0}+\Delta J\left(\tilde{V}_{l i}\right)$, therefore the sensitivity of the objective function $J$ can be written as

$$
\begin{aligned}
& \frac{\partial J}{\partial \tilde{V}_{1 i}}=\frac{\partial \Delta L}{\partial \tilde{V}_{1 i}}=\sum_{k=1}^{K}\left(-\int_{\varphi_{1}=0}\left(1-H\left(\varphi_{2}\right)\right)\left(-\frac{1}{2}{ }^{k} \Delta \boldsymbol{\varepsilon}_{{ }^{k} \Delta \mathbf{u}}+{ }^{k} \boldsymbol{\varepsilon}_{{ } \mathbf{w}}\right)^{\mathrm{T}} \mathbf{D}_{1}{ }^{k} \Delta \boldsymbol{\varepsilon}_{{ }^{k} \Delta \mathbf{u}} N_{i} \mathrm{~d} s\right) \\
& \frac{\partial J}{\partial \tilde{V}_{2 i}}=\frac{\partial \Delta L}{\partial \tilde{V}_{2 i}}=\sum_{k=1}^{K}\left(\int_{\varphi_{2}=0}-\left(-\frac{1}{2}{ }^{k} \Delta \boldsymbol{\varepsilon}_{{ } \Delta \mathbf{u}}+{ }^{k} \boldsymbol{\varepsilon}_{k_{\mathbf{w}}}\right)^{\mathrm{T}}\left(-H\left(\varphi_{1}\right) \mathbf{D}_{1}+\mathbf{D}_{2}\right)^{k} \Delta \boldsymbol{\varepsilon}_{{ }^{k} \Delta \mathbf{u}} N_{i} \mathrm{~d} s\right. \\
& \left.-\int_{\varphi_{2}=0} \kappa\left(\frac{1}{2}{ }^{k} \Delta \mathbf{u}_{\mathrm{disc}}-{ }^{k} \mathbf{w}_{\mathrm{disc}}\right)^{\mathrm{T}} \mathbf{G}^{\mathrm{T}}{ }^{k} \mathbf{T} \mathbf{G}^{k} \Delta \mathbf{u}_{\mathrm{disc}} H\left(\varphi_{1}\right) N_{i} \mathrm{~d} s\right) \\
& +b \int_{\varphi_{2}=0} \kappa H\left(\varphi_{1}\right) N_{i} \mathrm{~d} s
\end{aligned}
$$

Similarly, the change of the volume fraction constraints due to the perturbation caused by the velocities $V_{1 \mathrm{n}}$ and $V_{2 \mathrm{n}}$ can be written as

$$
\begin{gathered}
\Delta g_{1}=\left(\int_{\varphi_{1}=0}-\left(1-H\left(\varphi_{2}\right)\right) V_{1 \mathrm{n}} \mathrm{d} s+\int_{\varphi_{2}=0} H\left(\varphi_{1}\right) V_{2 \mathrm{n}} \mathrm{d} s\right) / \mathrm{V}_{\mathrm{D}}, \\
\Delta g_{2}=\left(\int_{\varphi_{2}=0}-V_{2 \mathrm{n}} \mathrm{d} s\right) / \mathrm{V}_{\mathrm{D}},
\end{gathered}
$$

in which $V_{D}$ is the volume of the design domain. Again, by substituting Eq. (24) into Eqs. (41) and (42), and re-writing $g_{1}$ and $g_{2}$ as $g_{1}=g_{10}+\Delta g_{1}\left(\tilde{V}_{l i}\right)$ and $g_{2}=g_{20}+\Delta g_{2}\left(\tilde{V}_{l i}\right)$ where $g_{10}$ and $g_{20}$ respectively denote current values of the volume fraction constraints for material 1 and material 2, the sensitivity of the volume fraction can be derived as 


$$
\begin{gathered}
\frac{\partial g_{1}}{\partial \tilde{V}_{1 i}}=\left(\int_{\varphi_{1}=0}-\left(1-H\left(\varphi_{2}\right)\right) N_{i} \mathrm{~d} s\right) / \mathrm{V}_{\mathrm{D}} \\
\frac{\partial g_{1}}{\partial \tilde{V}_{2 i}}=\left(\int_{\varphi_{2}=0} H\left(\varphi_{1}\right) N_{i} \mathrm{~d} s\right) / \mathrm{V}_{\mathrm{D}} \\
\frac{\partial g_{2}}{\partial \tilde{V}_{2 i}}=\left(\int_{\varphi_{2}=0}-N_{i} \mathrm{~d} s\right) / \mathrm{V}_{\mathrm{D}}
\end{gathered}
$$

As mentioned in Section 2.3, the zero level sets of $\varphi_{1}$ and $\varphi_{2}$ are found and approximated by a series of line segments. Hence, the sensitivities in Eqs. (39), (40), (43)-(45), are all calculated as line integrals on them.

In this study, we optimize the structural boundary velocities with the mathematical programming method MMA instead of the steepest descent direction-based searching used in the standard implementation of level set method. It is noted that a similar strategy was also used by Dunning and Kim in [40] in the topology optimization of single-material structures. In their work, the velocities of the level set function are defined on the intersection points of the zero level set and the element edges, and their optimal values are obtained with the sequential linear programming method.

\section{Numerical examples}

In all the numerical examples, the design domain is discretized with a structured mesh of plain-strain square elements with the size of 10 , and the level set points coincide with the finite element nodes. The properties of material 1 (a soft material) and material 2 (a stiff material) are $E_{1}=30000, E_{2}=60000$ and $v_{1}=v_{2}=0.15$; the void phase is modeled with the property of $E_{3}=E_{1} \times 10^{-6}$ and $v_{3}=0.15$. The diffusion coefficient $c$ in the Hamilton-Jacobi equation is 0.02 .

\subsection{Design of a simply-supported beam}


We first consider the optimization design of a simply-supported beam, as depicted in Fig. 8 . A prescribed displacement of $d_{\mathrm{P}}=0.04$ is imposed on the middle of the bottom edge. The parameters in the cohesive constitutive law are given as follows: the fracture energy $G_{\mathrm{f}}=0.05$, the maximum traction $\sigma_{\mathrm{c}}=1$, the separation of the interface corresponding to $\sigma_{\mathrm{c}}$ is $\delta_{\mathrm{c}}=G_{\mathrm{f}} /\left(e \sigma_{\mathrm{c}}\right)=0.0184$, the weight of the sliding opening displacement is $\beta=2$, and the constant compression stiffness is $d_{\mathrm{n}}=10^{8}$. These parameter values can be found in [28]. The nonlinear finite element analysis is divided equally into 20 incremental steps.

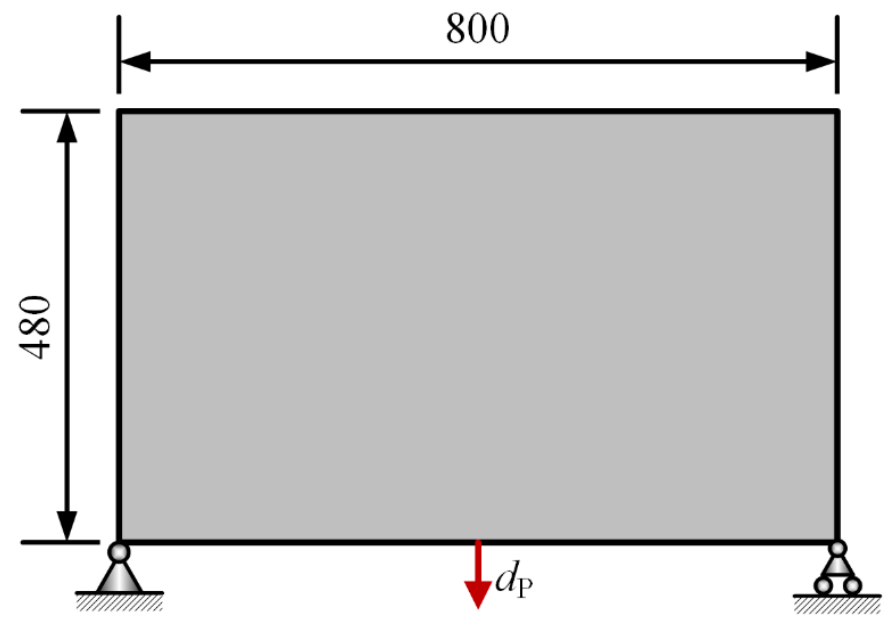

Fig. 8 Design domain of the simply-supported beam

The initial design of the structure is shown in Fig. 9. The green color represents material 1 (the soft material), and the blue color denotes material 2 (the stiff material). Here, the soft material $\left(\varphi_{1}>0, \varphi_{2}<0\right)$ is attached to the inside of the stiff material $\left(\varphi_{2}>0\right)$ to set up the initial interfaces $\left(\varphi_{1}>0, \varphi_{2}=0\right)$. In this specific design problem, when starting from a design domain with uniformly distributed holes, subtle interface evolutions confined in the vicinity of the concentrated loading point and supporting points (stress concentration regions) may slow down final convergence of the optimization process, although they would not affect the overall load transmission path. Therefore, in the mentioned initial design, the regions near the loading 
and supporting points are considered as non-design regions and filled with the stiff material. Our numerical results show that starting from such an initial design helps to improve convergence. The given volume fractions of the soft material and the stiff material are 0.1 and 0.2 , respectively. The weighting factor $b$ of the length term in the objective function is set to be 0.1 .

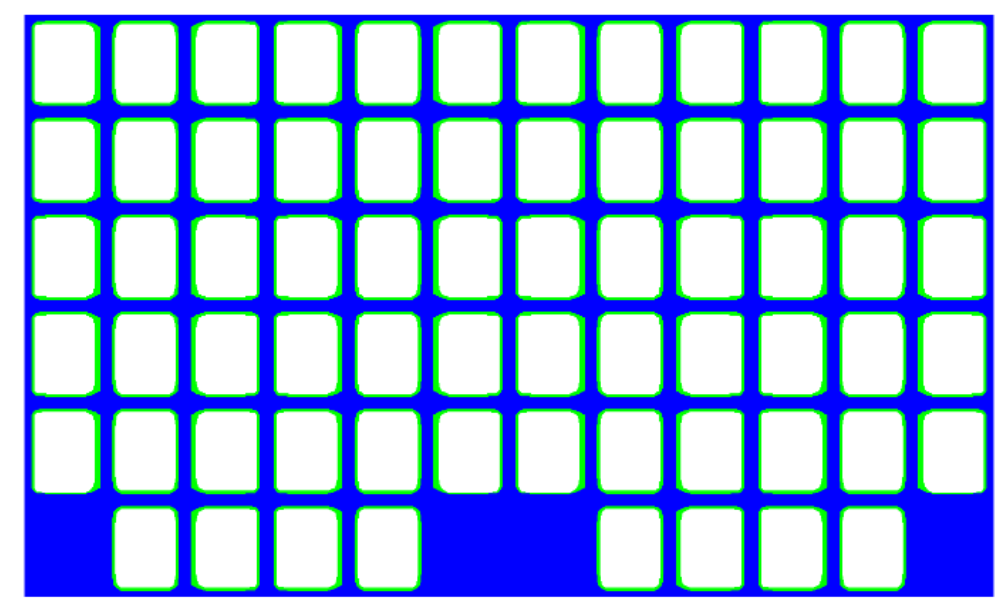

Fig. 9 Initial design of the simply-supported beam

Using the present method, the optimization process converged after 493 iterations. The optimal topology is shown in Fig. 10 (a), and the corresponding negative stored energy decreases from -0.3777 of the initial design to -1.027 in this final optimal design. As can be seen in Fig. 10 (a), the soft material appears in the arch part of the structure, while the stiff material forms the links between the arch and the loading point. More importantly, all the bonding interfaces locate in the regions under compression. This is intuitively reasonable because such a structural layout avoids opening of the bonding interfaces and thus improves the structural stiffness.

For comparison, the optimal design under the assumption of perfect bonding is also obtained (without enrichment shape functions for material interfaces) and given in Fig. 10 (b), and its negative stored energy is predicted to be -1.128 under the perfect bonding assumption. In Fig. 10 (b), the soft material and the interfaces appear in the bars connecting the loading point and 
the arch part. Clearly, the material interfaces in the two inner bar members are subject to tension under the considered loading condition. In contrast to the optimal design considering interface effect, such a design will be strongly affected by possible opening of the material interface, which is yet not considered in the conventional topology optimization of multimaterial structures.

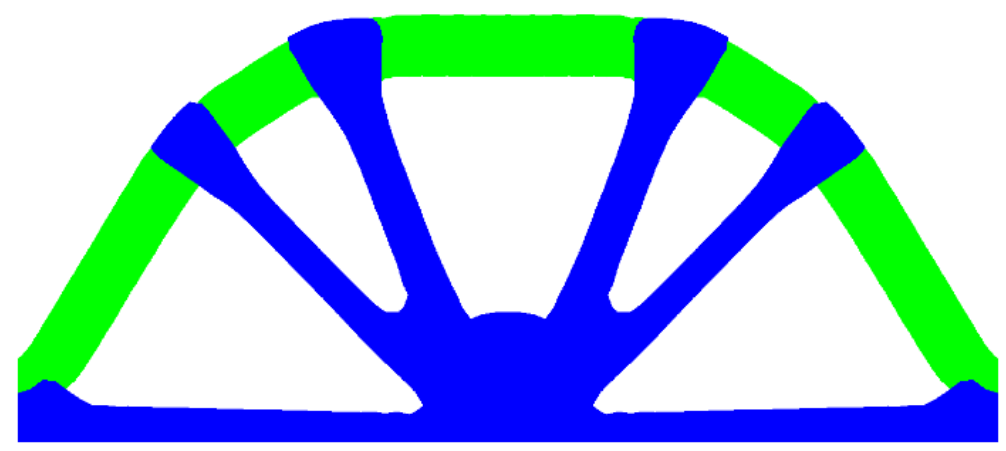

(a)

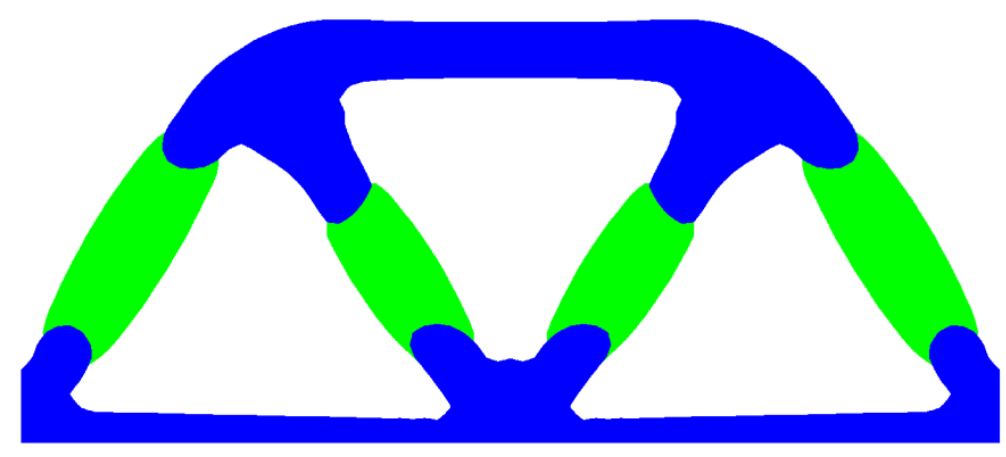

(b)

Fig. 10 Optimal designs (a) with bonding interface; (b) under assumption of perfect bonding

Hereafter in this numerical example, for comparison the design considering perfect bonding interface is re-analyzed with the cohesive model for capturing the material interfaces effects. To further compare the two optimal designs, the contours of the first principal stress (at the centroid points of the elements) are shown in Fig. 11. In the figures, the structural boundaries are depicted in white color and the interfaces in black color. As can be seen in Fig. 11 (a), the first principal stress level is very low in the arch part, implying that the arch part and all the 
material interfaces mainly sustains compression forces. On the contrary, Fig. 11 (b) indicates that some material interfaces are subjected to relatively high tension forces. This tendency can be also observed in the deformation plots shown in Fig. 12. Clearly, obvious debonding is seen in the conventional design, while this is not the case for the optimal design obtained with the proposed formulation.

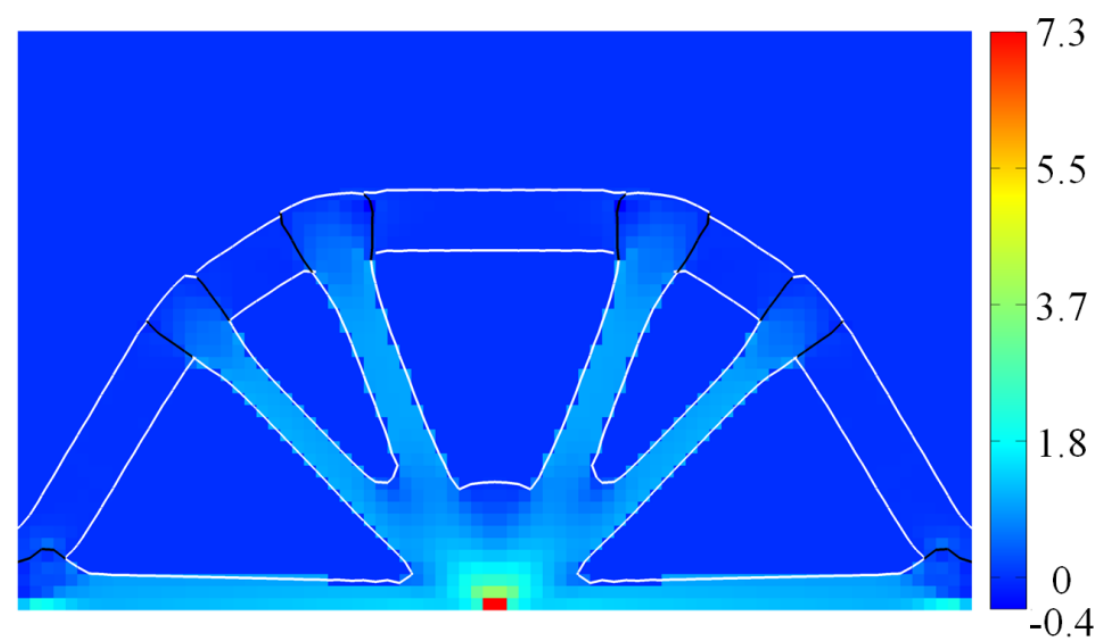

(a)

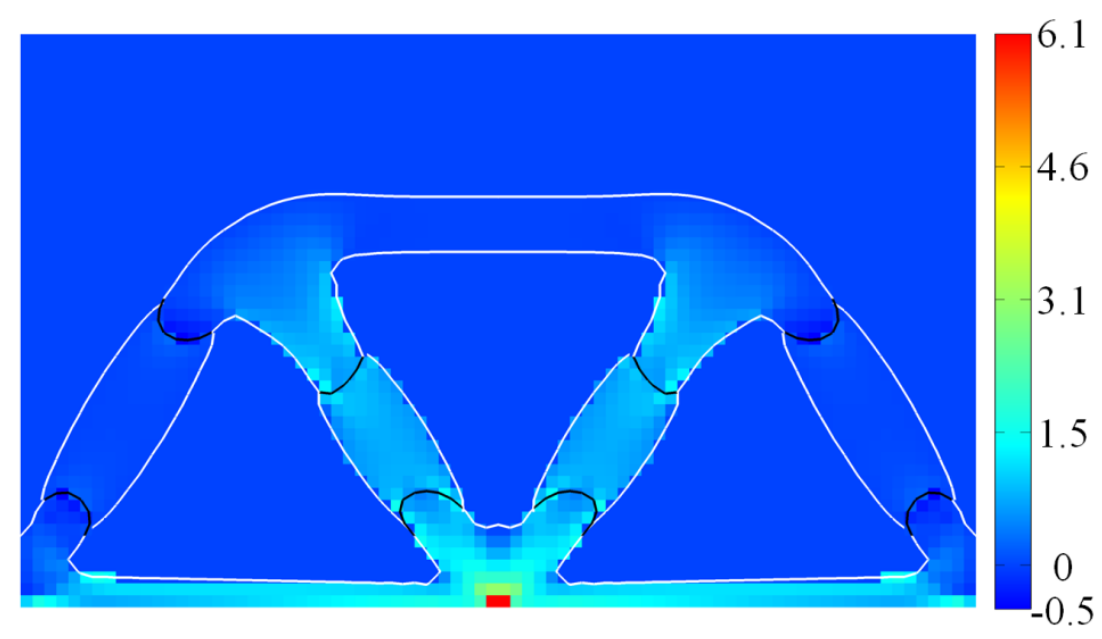

(b)

Fig. 11 First principal stress distribution of optimal designs (a) with bonding interface; (b) under assumption of perfect bonding 


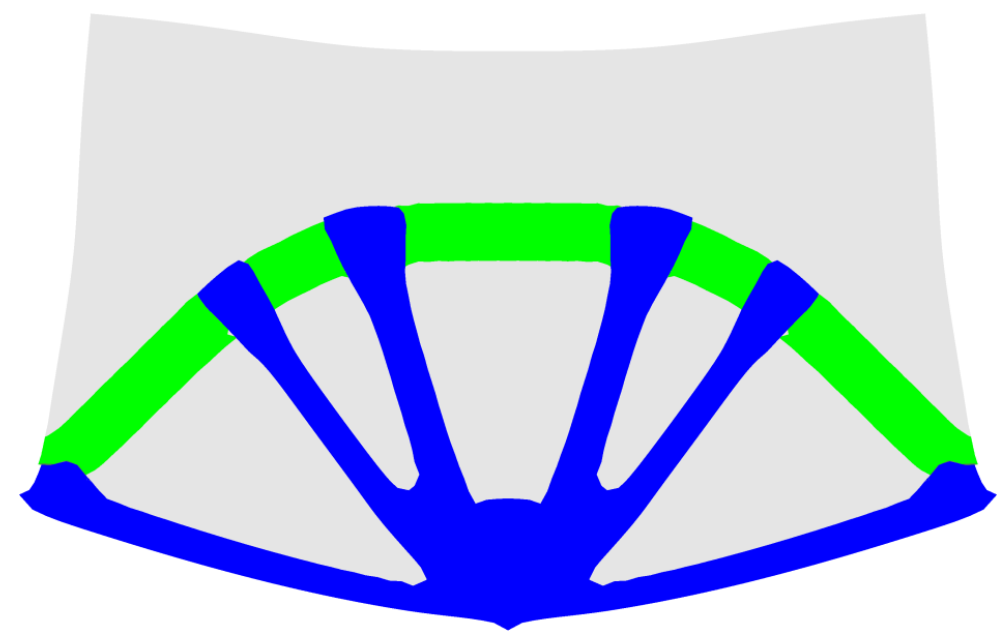

(a)

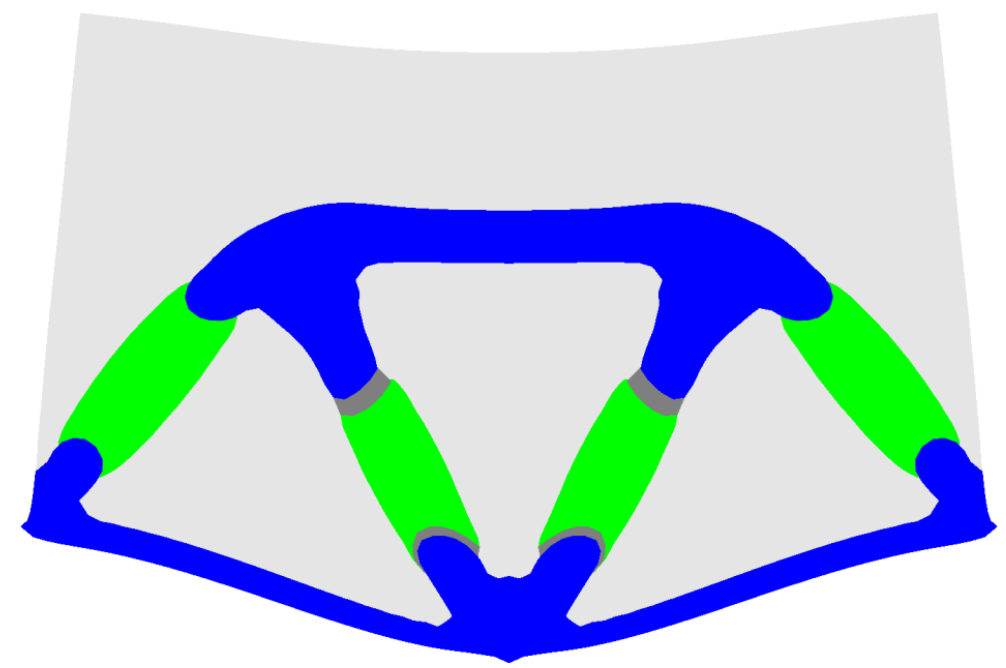

(b)

Fig. 12 Deformation plots (scale factor: 3000) of optimal designs (a) with bonding interface; (b) under assumption of perfect bonding

The iteration history of the optimization process is plotted in Fig. 13. The local fluctuation of the structural stored energy and the material volume constraints may be caused by the nonconvex and nonlinear nature of the considered topology optimization problem. 


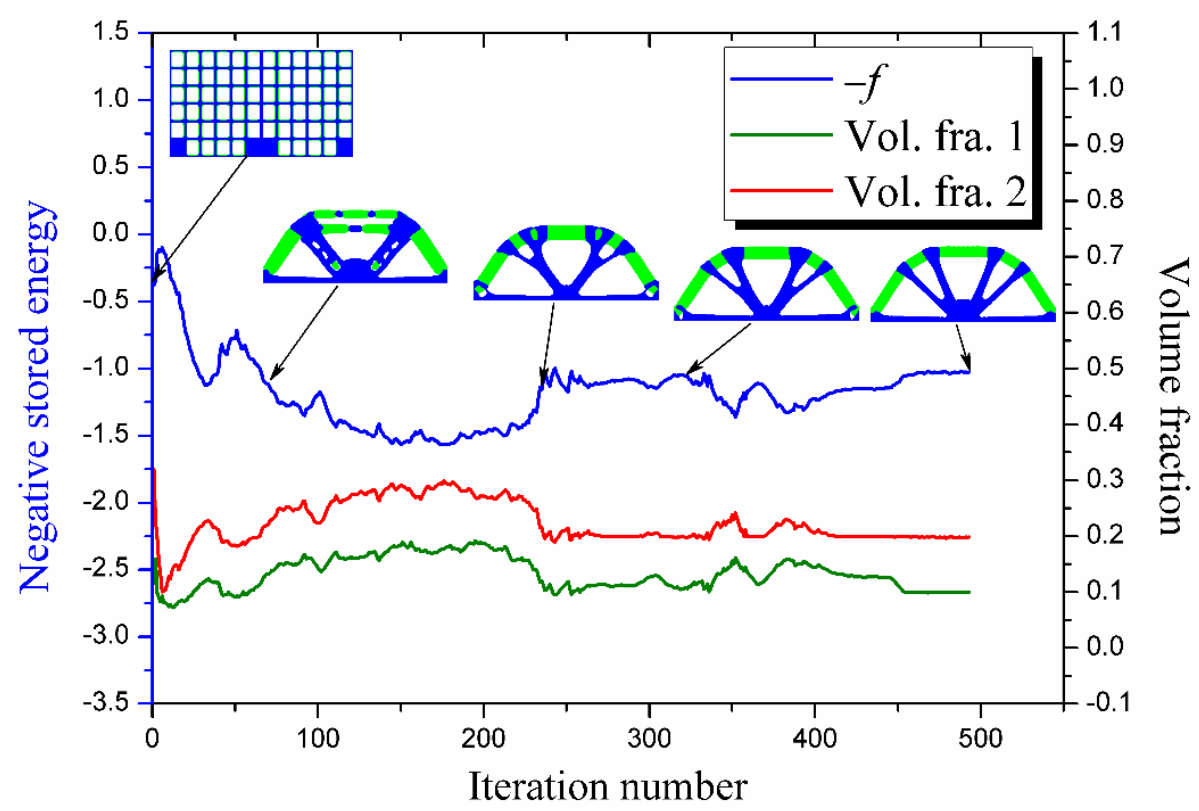

Fig. 13 Iteration history for the simply supported beam

\subsection{Design of a cantilever beam}

The second example is the topological design of a cantilever beam, as shown in Fig. 14. A prescribed displacement of $d_{\mathrm{P}}=0.04$ is applied to the middle point of the free end. In the nonlinear finite element analysis, this non-homogeneous Dirichlet boundary condition is gradually imposed with 20 incremental loading steps. The constraints of volume fraction for the soft and stiff materials are given as 0.25 and 0.3 , respectively. The initial design is depicted in Fig. 15.

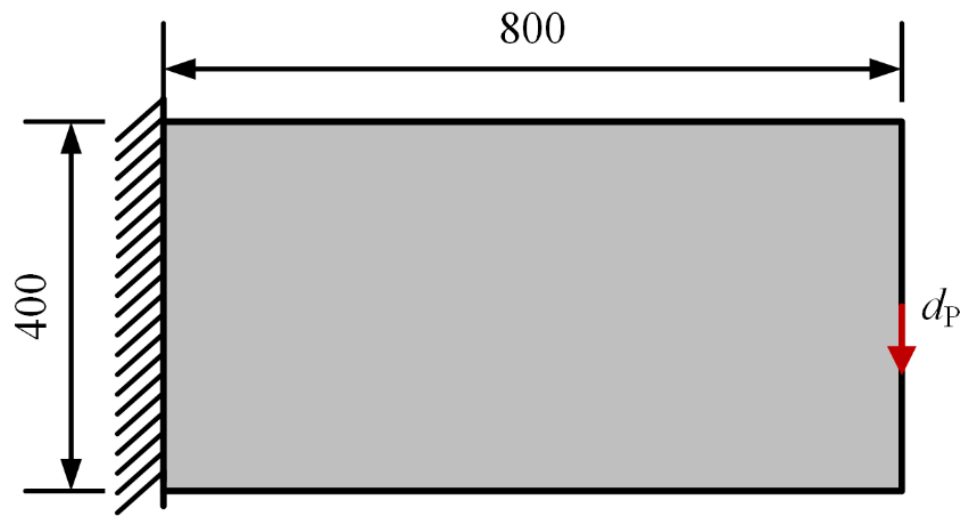

Fig. 14 Design domain of the cantilever beam 


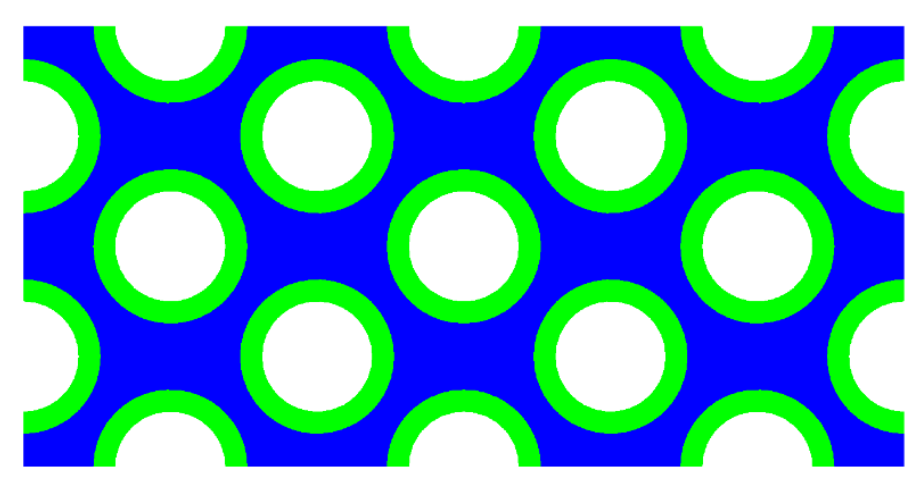

Fig. 15 Initial design of the cantilever beam

To study the interface effect, we considered different values of the maximum traction in the cohesive constitutive law, namely $\sigma_{\mathrm{c}}=1,10^{2}$ and $10^{4}$, while still using the mentioned values of other interface parameters in the first example. The weight factors for the cases of interface property of $\sigma_{\mathrm{c}}=1,10^{2}$ and $10^{4}$ are $b=0.08,0.02$ and 0.01 , respectively. For the purpose of comparison, the optimal design without consideration of bonding interface is also obtained. These optimal results are shown in Fig. 16.

From Figs. 16 (a)-(c), it is seen that the optimal designs are asymmetric and their layouts depend on the interface parameters. To be more specific, in Figs. 16 (a) and (b), the stiff material forms independent structural members. In Figs. 16 (c) and (d), the materials are relatively scattered, resulting in more material interfaces in the load transmission path. This is natural because in each of these three designs the interface has different load bearing capacity. It is also noted from Fig. 16 (d) that when the interface is treated as a perfectly bonded one, the optimal topology becomes symmetry.

The negative stored energy for the four optimal designs in Figs. 16 (a)-(d) are $-f=-0.628,-0.691,-0.691$ and -0.727 , respectively. It is seen that the structural stiffness generally increases with the interface parameter $\sigma_{\mathrm{c}}$. Particularly, when the interface is perfectly bonded, the optimal design achieves the maximum stiffness. 


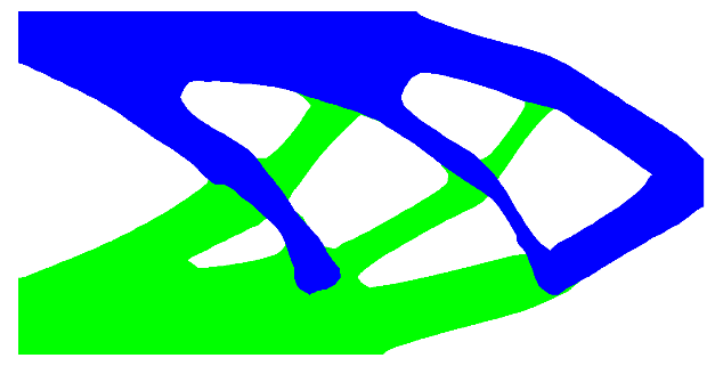

(a)

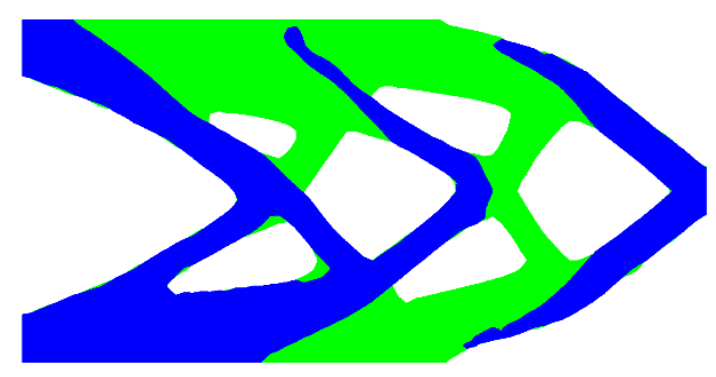

(c)

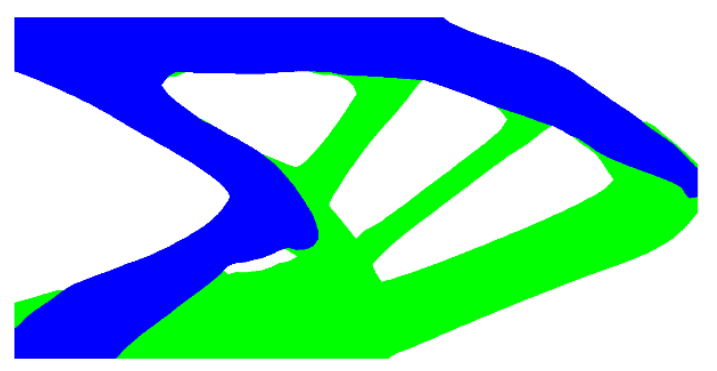

(b)

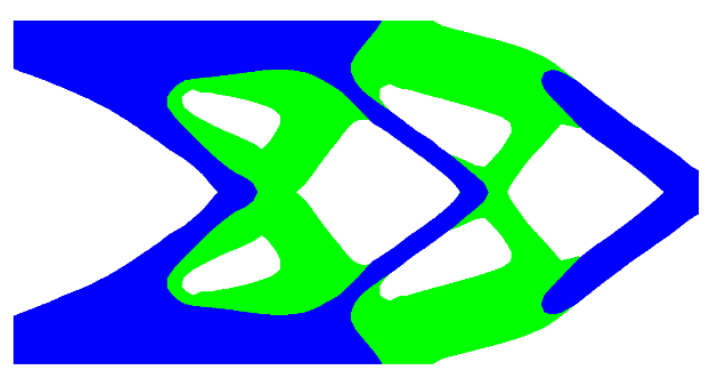

(d)

Fig. 16 Optimal designs obtained with different interface properties (a) $\sigma_{c}=1$; (b) $\sigma_{\mathrm{c}}=10^{2} ;(\mathrm{c}) \sigma_{\mathrm{c}}=10^{4} ;(\mathrm{d})$ perfect bonding

To verify the optimal designs, the first principal stress distributions of the optimal designs are shown in Fig. 17. Again, in the following comparisons, the response of the design under the perfect bonding assumption is obtained through re-analysis with the cohesive model $\left(\sigma_{\mathrm{c}}=1\right)$. In Figs. 17 (a) and (b), it is seen that the first principal stress is very low at the material interfaces and the stiff material mainly bears the tensile force. In Fig. 17 (c), as the interface has a larger load bearing capability, some of the material interfaces are exposed to a relatively high level of tensile force. Since the design in Fig. 17 (d) is initially obtained with the perfect bonding assumption, the first principal stresses at the interfaces in the upper part of the design are relatively high. 


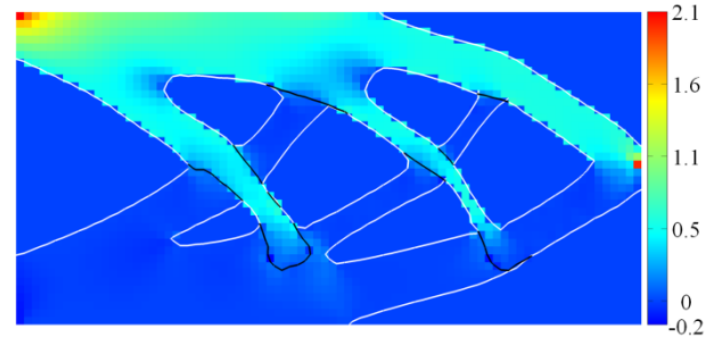

(a)

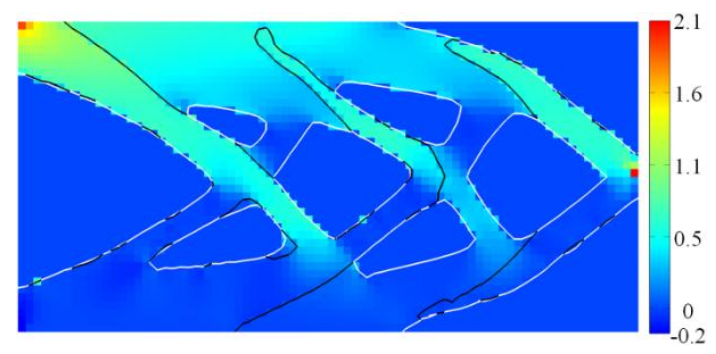

(c)

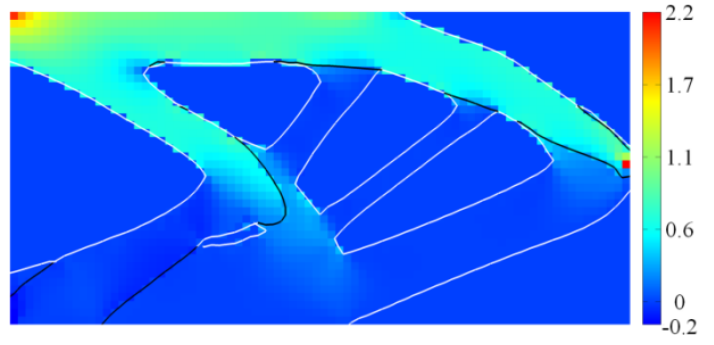

(b)

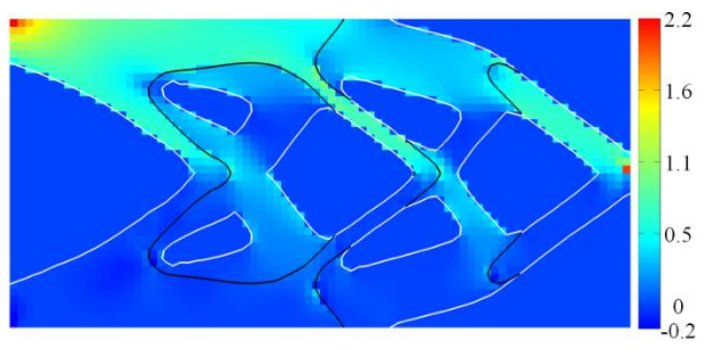

(d)

Fig. 17 First principal stress distributions of optimal designs obtained with different interface properties: (a) $\sigma_{\mathrm{c}}=1$; (b) $\sigma_{\mathrm{c}}=10^{2}$; (c) $\sigma_{\mathrm{c}}=10^{4}$; (d) perfect bonding

The deformation plots of these optimal designs are depicted in Fig. 18. It is seen in Figs. 18 (a)-(c) that in the designs obtained with the proposed method, the bonding interfaces have no distinct separation. However, as shown in Fig. 18 (d), in the design obtained without consideration of the interface behavior, certain interfaces cannot sustain the tensile and shear force and thus exhibit a relatively large separation.

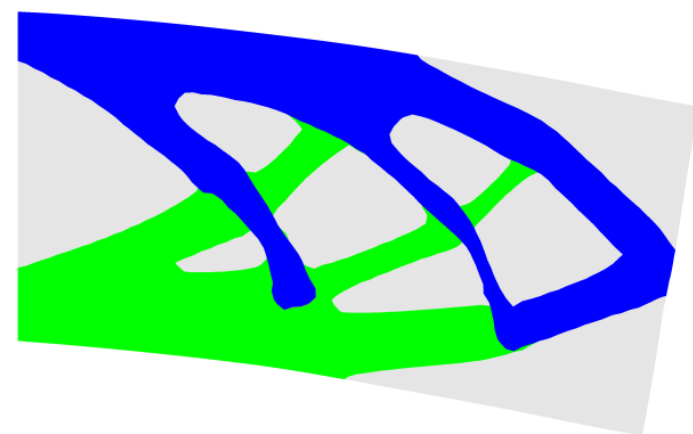

(a)

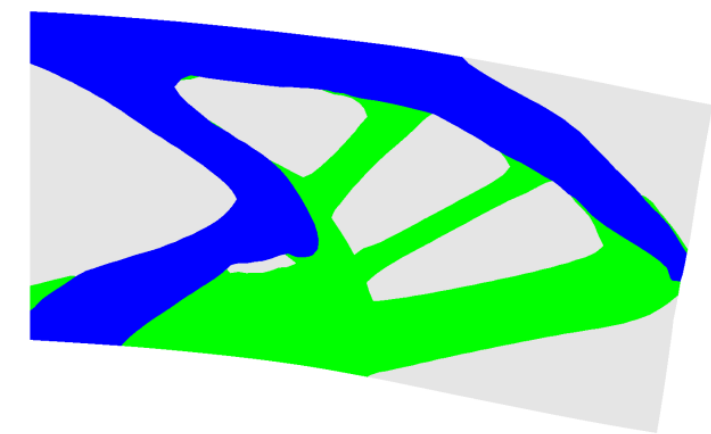

(b) 


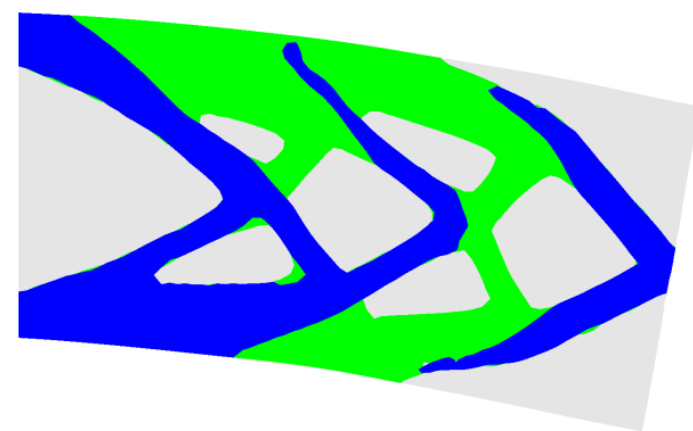

(c)

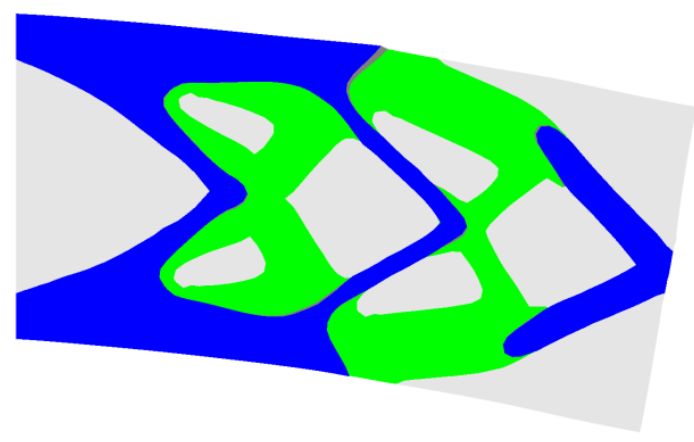

(d)

Fig. 18 Deformation plots (scale factor: 3000) of the designs obtained with different interface properties (a) $\sigma_{\mathrm{c}}=1$; (b) $\sigma_{\mathrm{c}}=10^{2}$; (c) $\sigma_{\mathrm{c}}=10^{4}$; (d) perfect bonding

To study the effects of the length term in the objective function, we re-ran the optimization process $\left(\sigma_{\mathrm{c}}=1\right.$ ) with different weight factors $b=0.04$ and 0.14 . The optimized topologies are given in Fig. 19. The length of the bonding interface for the designs in Fig. 19 (a) and (b) are 657 and 451, respectively. As can be seen, with different weight factor $b$, the optimization process may converge to different local optima.

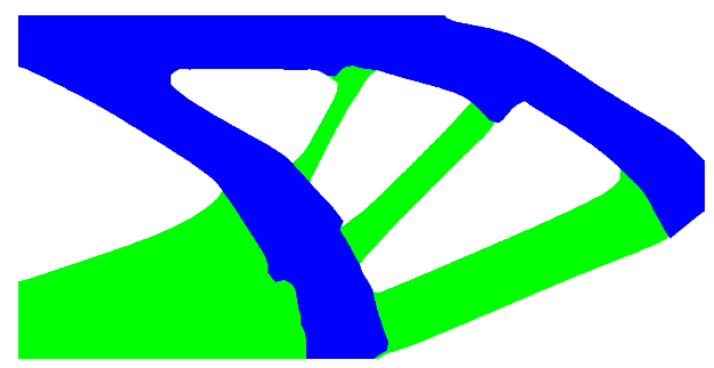

(a)

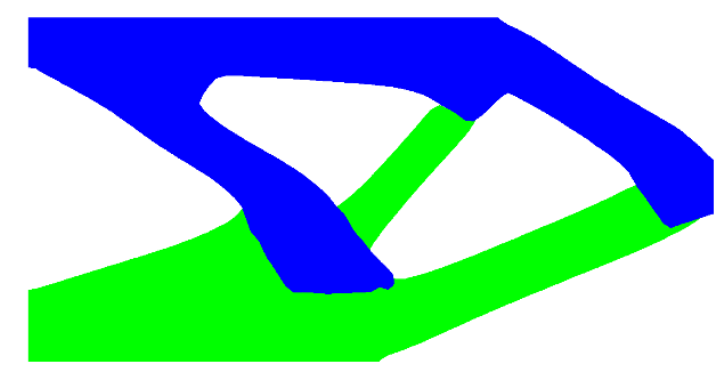

(b)

Fig. 19 Optimal designs obtained with the interface property that $\sigma_{\mathrm{c}}=1$ and different weigh factors of the length term (a) $b=0.04$; (b) $b=0.14$

The iteration history of the optimization process for the design in Fig. 16 (a) is shown in Fig. 
20. The iteration ends after 163 iterations. The negative stored energy decreases from -0.2615 of the initial design to -0.6278 of the optimal design.

The volume constraints are active $\left(f_{\mathrm{V}_{1}}=f_{\overline{\mathrm{V}}_{1}}, f_{\mathrm{V}_{2}}=f_{\overline{\mathrm{V}}_{2}}\right)$ for all the optimal designs in Figs. 16 (a)-(d) and Figs. 19 (a)-(b).

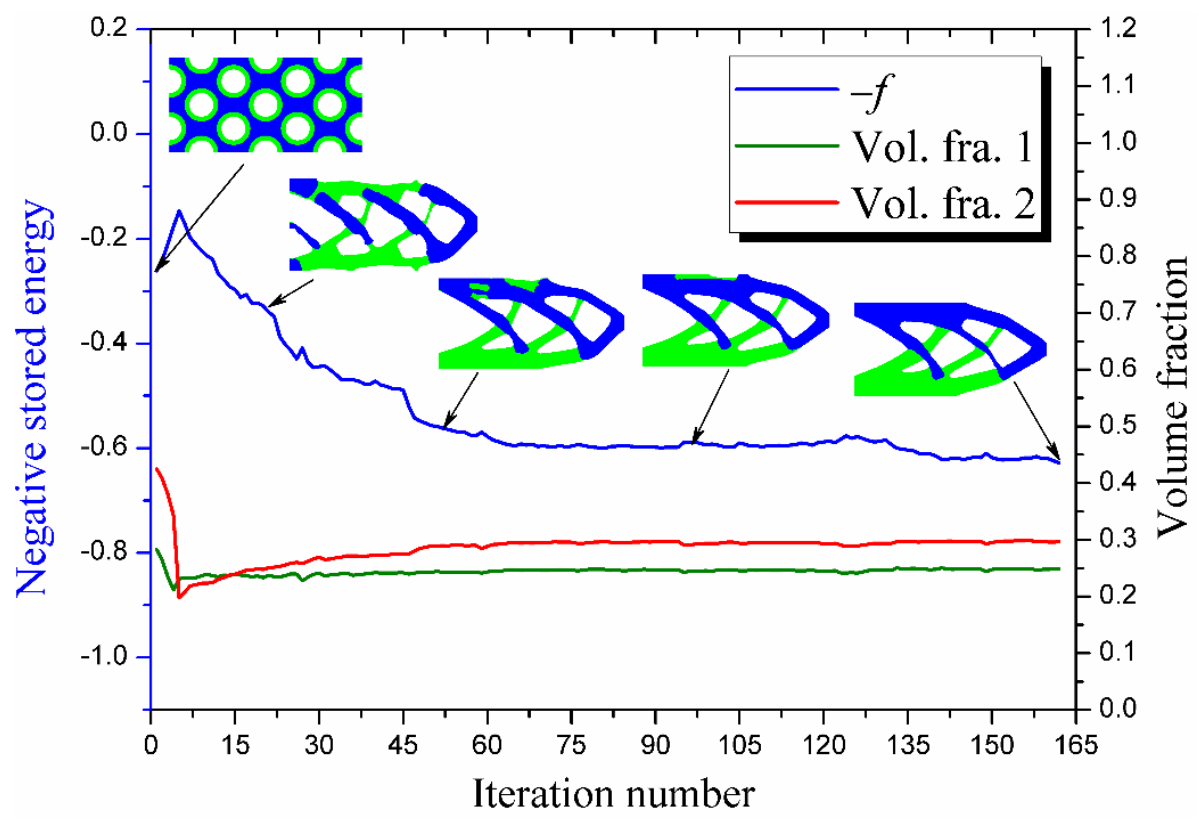

Fig. 20 Iteration history for the cantilever beam

\section{Conclusions}

Interface behaviors play a key role in the stiffness and strength of an optimal design composed of multi-phase materials. This study proposed a new framework to take into account the material interface behaviors in a maximum stiffness topology optimization problem with multi-phase materials. The color level set method is employed to describe the topology of the design and to define the positions of the bonding interfaces. The response of the interfaces is computed with the combination of the XFEM and a cohesive model. This modeling technique provides a more realistic description of the interface behavior, which accounts for the interface opening, and at the same time avoids the difficulties of re-meshing for tracking the moving interface during the optimization process. The velocities of the level set points as defined as the 
design variables. In conjunction with the adjoint sensitivity analysis, these design variables are obtained with the mathematical programming method MMA and then used to interpolate the velocities on the boundary points of the design. The boundary velocities are extrapolated to the whole design domain and the Hamilton-Jacobi equation is employed to advance the level set functions and update the design. In the framework of level set method, this optimization technique can handle multi-constraints easily while still preserving the advantages of conventional level set methods, and thus can be employed in topology optimization problem with even more material phases.

The numerical examples demonstrate validity of the proposed method. The need to consider the interfaces behaviors is highlighted through comparisons between the designs obtained with the proposed method and those with perfect bonding assumption. It is also found that the interfaces mainly undergo compression in the optimized structures.

Obviously, the inherent non-convexity and nonlinearity exhibited by the considered topology optimization problem may cause certain fluctuations of the objective functions and constraints during the optimization iterations. This can be alleviated by employing some stability control (e.g. adaptive moving limit) or feature size control techniques. However, it does not fall within the main topic of this study. It may be also interesting to extend the proposed method to the design of microstructural composite materials.

\section{Acknowledgements}

The supports of the National Science Foundation of China (11425207, U1508209 and 91530110) are gratefully acknowledged.

\section{References}

[1] M.P. Bendsøe, N. Kikuchi, Generating optimal topologies in structural design using a homogenization method, Computer methods in applied mechanics and engineering, 71 (1988) 197-224.

[2] M.P. Bendsøe, Optimal shape design as a material distribution problem, Structural optimization, 1 (1989) 193-202. 
[3] G.I. Rozvany, M. Zhou, T. Birker, Generalized shape optimization without homogenization, Structural optimization, 4 (1992) 250-252.

[4] M.P. Bendsøe, O. Sigmund, Material interpolation schemes in topology optimization, Archive of applied mechanics, 69 (1999) 635-654.

[5] M.Y. Wang, X. Wang, D. Guo, A level set method for structural topology optimization, Computer methods in applied mechanics and engineering, 192 (2003) 227-246.

[6] G. Allaire, F. Jouve, A.-M. Toader, Structural optimization using sensitivity analysis and a level-set method, Journal of computational physics, 194 (2004) 363-393.

[7] Y. Xie, G.P. Steven, A simple evolutionary procedure for structural optimization, Computers \& structures, 49 (1993) 885-896.

[8] G.I. Rozvany, A critical review of established methods of structural topology optimization, Structural and Multidisciplinary Optimization, 37 (2009) 217-237.

[9] N.P. van Dijk, K. Maute, M. Langelaar, F. Van Keulen, Level-set methods for structural topology optimization: a review, Structural and Multidisciplinary Optimization, 48 (2013) 437-472.

[10] J.D. Deaton, R.V. Grandhi, A survey of structural and multidisciplinary continuum topology optimization: post 2000, Structural and Multidisciplinary Optimization, 49 (2014) 1-38.

[11] J.-H. Zhu, W.-H. Zhang, L. Xia, Topology optimization in aircraft and aerospace structures design, Archives of Computational Methods in Engineering, (2015) 1-28.

[12] O. Sigmund, S. Torquato, Design of materials with extreme thermal expansion using a three-phase topology optimization method, in: Smart Structures and Materials' 97, International Society for Optics and Photonics, 1997, pp. 52-60.

[13] L.V. Gibiansky, O. Sigmund, Multiphase composites with extremal bulk modulus, Journal of the Mechanics and Physics of Solids, 48 (2000) 461-498.

[14] J. Stegmann, E. Lund, Discrete material optimization of general composite shell structures, International Journal for Numerical Methods in Engineering, 62 (2005) 2009-2027.

[15] C.F. Hvejsel, E. Lund, Material interpolation schemes for unified topology and multi-material optimization, Structural and Multidisciplinary Optimization, 43 (2011) 811-825.

[16] M. Bruyneel, SFP - a new parameterization based on shape functions for optimal material selection: application to conventional composite plies, Structural and Multidisciplinary Optimization, 43 (2011) 17-27.

[17] T. Gao, W. Zhang, P. Duysinx, A bi-value coding parameterization scheme for the discrete optimal orientation design of the composite laminate, International Journal for Numerical Methods in Engineering, 91 (2012) 98-114.

[18] L. Yin, G. Ananthasuresh, Topology optimization of compliant mechanisms with multiple materials using a peak function material interpolation scheme, Structural and Multidisciplinary Optimization, 23 (2001) 49-62.

[19] A. Radman, X. Huang, Y. Xie, Topological design of microstructures of multiphase materials for maximum stiffness or thermal conductivity, Computational Materials Science, 91 (2014) 266-273. 
[20] M.Y. Wang, X. Wang, "Color" level sets: a multi-phase method for structural topology optimization with multiple materials, Computer Methods in Applied Mechanics and Engineering, 193 (2004) 469-496.

[21] M.Y. Wang, S. Chen, X. Wang, Y. Mei, Design of multimaterial compliant mechanisms using level-set methods, Journal of Mechanical Design, 127 (2005) 941956.

[22] C. Zhuang, Z. Xiong, H. Ding, Topology optimization of multi-material for the heat conduction problem based on the level set method, Engineering Optimization, 42 (2010) 811-831.

[23] Y. Mei, X. Wang, A level set method for structural topology optimization and its applications, Advances in Engineering software, 35 (2004) 415-441.

[24] Y. Wang, Z. Luo, Z. Kang, N. Zhang, A multi-material level set-based topology and shape optimization method, Computer Methods in Applied Mechanics and Engineering, 283 (2015) 1570-1586.

[25] Z. Luo, L. Tong, J. Luo, P. Wei, M.Y. Wang, Design of piezoelectric actuators using a multiphase level set method of piecewise constants, Journal of Computational Physics, 228 (2009) 2643-2659.

[26] Y. LIU, D. MATSUNAKA, M. SHIMODA, Y. SHIBUTANI, Interface shape design of multi-material structures for delamination strength, Mechanical Engineering Journal, (2016).

[27] M. Lawry, K. Maute, Level set topology optimization of problems with sliding contact interfaces, Structural and Multidisciplinary Optimization, 52 (2015) 1107-1119.

[28] C.F. Hilchenbach, E. Ramm, Optimization of multiphase structures considering damage, Structural and Multidisciplinary Optimization, (2014) 1-14.

[29] K. Svanberg, The method of moving asymptotes- a new method for structural optimization, International journal for numerical methods in engineering, 24 (1987) 359-373.

[30] D. Adalsteinsson, J.A. Sethian, The fast construction of extension velocities in level set methods, Journal of Computational Physics, 148 (1999) 2-22.

[31] R. De Borst, M.A. Crisfield, J.J. Remmers, C.V. Verhoosel, Nonlinear finite element analysis of solids and structures, John Wiley \& Sons, 2012.

[32] G. Wells, L. Sluys, A new method for modelling cohesive cracks using finite elements, International Journal for Numerical Methods in Engineering, 50 (2001) 26672682.

[33] N. Moës, T. Belytschko, Extended finite element method for cohesive crack growth, Engineering fracture mechanics, 69 (2002) 813-833.

[34] M. Elices, G. Guinea, J. Gomez, J. Planas, The cohesive zone model: advantages, limitations and challenges, Engineering fracture mechanics, 69 (2002) 137-163.

[35] M. Ortiz, A. Pandolfi, Finite-deformation irreversible cohesive elements for threedimensional crack-propagation analysis, International Journal for Numerical Methods in Engineering, 44 (1999) 1267-1282.

[36] T.-P. Fries, T. Belytschko, The extended/generalized finite element method: an overview of the method and its applications, International Journal for Numerical Methods in Engineering, 84 (2010) 253-304. 
[37] J. Dolbow, T. Belytschko, A finite element method for crack growth without remeshing, Int. J. Numer. Meth. Eng, 46 (1999) 131-150.

[38] F. Niu, S. Xu, G. Cheng, A general formulation of structural topology optimization for maximizing structural stiffness, Structural and Multidisciplinary Optimization, 43 (2011) 561-572.

[39] T. Gao, W. Zhang, A mass constraint formulation for structural topology optimization with multiphase materials, International Journal for Numerical Methods in Engineering, 88 (2011) 774-796.

[40] P.D. Dunning, H.A. Kim, Introducing the sequential linear programming level-set method for topology optimization, Structural and Multidisciplinary Optimization, 51 (2014) 631-643. 